ARTICLE

https://doi.org/10.1038/s41467-019-11781-x

\title{
Wilm's tumor 1 promotes memory flexibility
}

Chiara Mariottini (10) ${ }^{1,2}$, Leonardo Munari (10 ${ }^{1}$, Ellen Gunzel ${ }^{1,2}$, Joseph M. Seco ${ }^{1}$, Nikos Tzavaras ${ }^{1}$, Jens Hansen (10) 1,2, Sarah A. Stern ${ }^{3,4}$, Virginia Gao ${ }^{3,4}$, Hossein Aleyasin³, Ali Sharma (1) 1, Evren U. Azeloglu (1) ${ }^{5}$, Georgia E. Hodes (10 ${ }^{3}$, Scott J. Russo (10 ${ }^{3}$, Vicki Huff ${ }^{6}$, Marc R. Birtwistle ${ }^{1,2}$, Robert D. Blitzer ${ }^{1}$, Cristina M. Alberini ${ }^{3,4} \&$ Ravi lyengar ${ }^{1,2}$

Under physiological conditions, strength and persistence of memory must be regulated in order to produce behavioral flexibility. In fact, impairments in memory flexibility are associated with pathologies such as post-traumatic stress disorder or autism; however, the underlying mechanisms that enable memory flexibility are still poorly understood. Here, we identify transcriptional repressor Wilm's Tumor 1 (WT1) as a critical synaptic plasticity regulator that decreases memory strength, promoting memory flexibility. WT1 is activated in the hippocampus following induction of long-term potentiation (LTP) or learning. WT1 knockdown enhances CA1 neuronal excitability, LTP and long-term memory whereas its overexpression weakens memory retention. Moreover, forebrain WT1-deficient mice show deficits in both reversal, sequential learning tasks and contextual fear extinction, exhibiting impaired memory flexibility. We conclude that WT1 limits memory strength or promotes memory weakening, thus enabling memory flexibility, a process that is critical for learning from new experiences.

\footnotetext{
${ }^{1}$ Department of Pharmacological Sciences, Icahn School of Medicine at Mount Sinai, One Gustave Levy Place, New York 10029 NY, USA. ${ }^{2}$ Systems Biology Center, Icahn School of Medicine at Mount Sinai, One Gustave Levy Place, New York 10029 NY, USA. ${ }^{3}$ Department of Neuroscience, Icahn School of Medicine at Mount Sinai, One Gustave Levy Place, New York 10029 NY, USA. ${ }^{4}$ Center for Neural Science, New York University, New York 10003 NY, USA. ${ }^{5}$ Department of Medicine, Division of Nephrology, Icahn School of Medicine at Mount Sinai, One Gustave Levy Place, New York 10029 NY, USA.

${ }^{6}$ Department of Genetics, M.D. Anderson Cancer Center, University of Texas, Houston 77030 TX, USA. Correspondence and requests for materials should be addressed to C.M. (email: chiara.mariottini@mssm.edu) or to C.M.A. (email: ca60@nyu.edu) or to R.I. (email: ravi.iyengar@mssm.edu)
} 
earning produces long-term memory retention and storage by activating molecular mechanisms that consolidate and strengthen an initially labile experience representation. The process of memory strengthening must be regulated in order to remain within the physiological ranges; excessively weak or excessively strong memories are in fact maladaptive and pathological. Weak memories can result from impairments in any of several different processes-storage, retrieval, or consolidation (the stabilization process that forms long-term memories) or by an overactive forgetting process ${ }^{1-3}$. All these processes likely play important roles in memory disorders, in Alzheimer's disease, aging-related memory loss, and neurodevelopmental cognitive impairments. Conversely, an excessive memory consolidation, and/or impaired forgetting may produce excessively strong and inflexible memories, possibly leading to diseases such as posttraumatic stress disorder (PTSD), autism spectrum disorder, schizophrenia, and obsessive compulsive disorder (OCD). Therefore, the ability to regulate the intensity of memory consolidation and strengthening is of great importance for adaptive behaviors and mental health.

The biological mechanisms required for promoting memory consolidation and strengthening have been investigated in many species and types of memory, identifying roles for a variety of signaling networks ${ }^{4,5}$, transcription factors ${ }^{6,7}$, and epigenetic changes $^{8}$. However, little is known about mechanisms that reduce memory consolidation and strengthening in order to enable behavioral flexibility. A key question is whether consolidated memories are weakened through a passive decay process, and/or by a learning-induced, active mechanisms that serves to promote memory flexibility. In other words, do signaling pathways that are activated during experience not only support consolidation, but also include counteracting molecular regulators that can decrease memory strength and favor forgetting ${ }^{3}$, such as the Rho family of GTPases signaling G proteins (Rac) ${ }^{9,10}$, scribble scaffolds ${ }^{11}$, DAMB dopamine receptors ${ }^{12}$, inhibition of AMPA receptor recycling $^{13}$, and neurogenesis ${ }^{14}$ ?

We therefore tested the hypothesis that memory flexibility results from an active process that occurs in parallel with memory consolidation and strengthening. If this is the case, then mechanisms enabling memory flexibility should be activated and/ or induced by learning.

Memory consolidation engages complex regulation of genes transcription activation and repression ${ }^{4}$. Whereas the role of transcription activators, such as members of the CREB, C/EBP, AP1, NFkB, Rel, Egr 1 and 2, and Nurr families have been more extensively documented as promoters of memory consolidation and strengthening $2,5,7,15-18$, less is known about the role of transcription repressors ${ }^{4,19,20}$. A few transcription repressors that directly bind to promoter/enhancer DNA sequences in memory formation have been documented: $\mathrm{CREB}^{21,22}, \mathrm{MeCP}^{23}$, DREAM (downstream regulatory element antagonistic modulator $)^{24}$, myocyte enhancer factor-2 (MEF2) ${ }^{25}$. The literature thus far suggests that induction of transcription activation correlates with memory strengthening, whereas induction of transcription repression correlates with memory weakening or forgetting $3,4,19,20$.

To search for transcription repressors of plasticity, we screened for transcription factors activated by induction of LTP at hippocampal excitatory synapses, a cellular model of learning and memory ${ }^{26}$. We identified Wilm's tumor 1 (WT1), a protein that is important for kidney and gonads development ${ }^{27}$. WT1 is a form of kidney cancer that primarily affects children ages $3-4$. Interestingly one of the health conditions due to Wt1 germline mutations is the WAGR syndrome, a disorder characterized by Wilm's Tumor (W), aniridia (A), genitourinary anomalies $(G)$ and mental retardation (R). Patients with WAGR syndrome have difficulties in learning, processing, and responding to information; they may develop behavioral and cognitive abnormalities such as anxiety, OCD, depression, attention deficit hyperactivity disorder, and autism ${ }^{28}$. While $W t 1$ gene has been well characterized for its role in kidney development and function, its role in the brain is not fully understood. WT1 has been linked to neurodegeneration associated with Alzheimer disease ${ }^{29}$, and a recent study has showed that during early neuronal development its transcriptional activity is repressed to allow normal neuronal differentiation $^{30}$. In this study, we use different types of genetic and molecular manipulation to investigate the functional role of WT1 in memory consolidation and strengthening and its ability to regulate memory flexibility and new learning. We also investigate WT1 hippocampal physiology by examining the effects of ablating WT1 on pyramidal cell excitability, synaptic plasticity, and regulation of entorhinal cortex-hippocampus circuitry. In addition, we identify numerous transcriptional targets of WT1 in the hippocampus and functionally characterize one of these genes in plasticity experiments. Our data indicate that the transcriptional repressor WT1 is a key regulator of synaptic plasticity, memory strength, and memory flexibility in the hippocampus.

\section{Results}

Learning-induced WT1 decreases memory strength. To identify transcription factors activated or induced by long-term plasticity, we employed a protein-DNA binding array on rat hippocampal slices in which long-term potentiation (LTP) was induced by strong high-frequency stimulation (Strong-HFS) of the Schaffer collaterals ${ }^{26}$. We identified nearly 40 transcription factors whose binding was increased (Fig. 1a and Supplementary Fig. 1a). One of these transcription factors, WT1, is a transcriptional repressor shown to be involved in regulating kidney development ${ }^{27}$ and in mRNA transport and translation in several cell lines ${ }^{31,32}$.

Strong-HFS as well as contextual fear conditioning (CFC) learning increased the binding of WT1 to its DNA consensus sequence in the hippocampus of rats (Fig. 1b, c), providing functional evidence for an active involvement of WT1 in these functions. Furthermore, we found independent evidence for WT1 activation in mRNA-seq experiments that identified increased expression of transcripts $90 \mathrm{~min}$ after LTP induction. Enrichment analysis of this transcriptomic data (see Supplementary Data 1 for complete list of differentially expressed transcripts) predicted WT1 as the second most likely candidate to regulate LTP-induced gene expression followed by members of the CREB family (ATF2 and ATF4) (Fig. 1d; see Supplementary Data 2 for predicted transcription factors analysis).

In addition both LTP induction-but not LTD-(LTP, Fig. 1e; LTD, Supplementary Fig. 2a) as well as contextual fear learning in two independent tasks, contextual fear conditioning (CFC, Fig. 1f; behavioral data shown in Supplementary Fig. 2b) and inhibitory avoidance (IA, Fig. 1g; for behavioral data see reference Taubenfeld et al. ${ }^{18}$ ), resulted in significant increases in the expression levels of WT1 protein within $30 \mathrm{~min}$. These data suggest that induction of WT1 is due to the learning process and not to the presentation of the aversive stimulus itself as we did not observe any significant change in WT1 expression using an unpaired CFC protocol (Supplementary Fig. 2c, d).

To determine the functional role of WT1 in memory formation, we knockdown WT1 protein expression using bilateral injections of antisense oligodeoxynucleotides (WT1-AS) into rat dorsal hippocampus (Fig. 2a) and tested the effect on memory retention using two different hippocampal tasks, one aversive (CFC) and one nonaversive (novel object location, NOL). As shown, WT1-AS compared with control scrambled oligodeoxynucleotides (SC-ODN) significantly decreased WT1 protein levels in dorsal hippocampus and resulted in a significantly enhanced CFC memory retention $24 \mathrm{~h}$ after training (Fig. 2a). Rats injected with either WT1-AS or SC-ODN did not differ in 
a

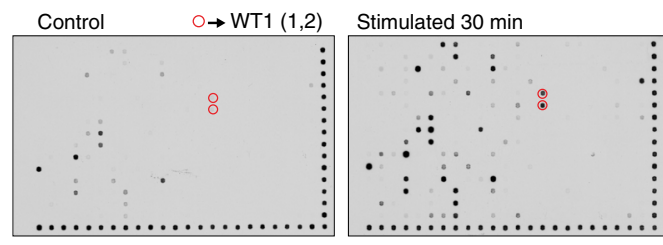

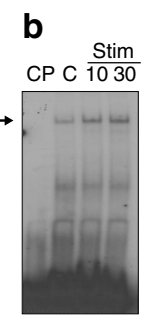

\section{C $\mathrm{CFC}(\min )$}

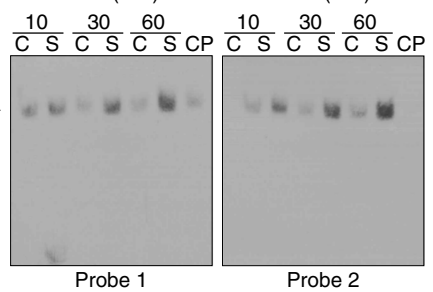

f $\mathrm{CFC}$

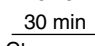

Ctx

only Paired
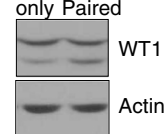

- Context only $n=4$

- Paired $n=5$

g IA

Trained

Naive $30 \mathrm{~min}$

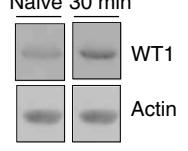

- Control $n=7$

- Stimulated $30 \min n=7$

- Naive $n=10$

- Trained $30 \min n=10$
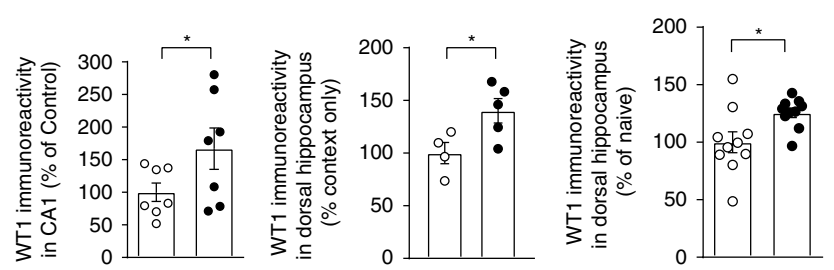

Fig. 1 WT1 expression and DNA-binding activity are induced by synaptic plasticity and learning. a Protein-DNA binding assay comparing rat hippocampal CA1 extracts from control tissue versus extracts obtained from tissue where LTP was induced. WT1 is circled in red; numbers in parentheses indicate two different DNA probes with WT1 consensus sites. b EMSA showing increased in vitro WT1 binding to a DNA consensus sequence (arrow indicates the WT1/DNA complex) 10 and 30 min after induction of LTP in hippocampal CA1 region (Stim) compared with unstimulated control (C). The specificity of DNA-protein binding was verified by incubation with excess unlabeled cold probe (CP). c EMSA showing increased WT1 binding to DNA (arrow indicates the WT1/DNA complex) at different time points after CFC (S, shocked group; C, context only controls). The specificity of DNA-protein binding was verified by incubation with excess unlabeled cold probe (CP). d Bar graph of the top ten transcription factors predicted to regulate gene expression profiles in rat tissue obtained 90 min after a stimulation that produced LTP. e Expression of WT1 was significantly increased in rat CA1 region 30 min after LTP induction (paired $t$ test: $\left.{ }^{*} p=0.0495\right)$. $\mathbf{f}$ WT1 expression in the dorsal hippocampus of rats trained in CFC (Paired) compared with non shocked rats (Ctx only) (unpaired $t$ test: $\left.{ }^{*} p=0.0385\right)$. $\mathbf{g}$ Expression of WT1 was significantly increased in the dorsal hippocampus of rats trained in an IA task. Protein expression was measured 30 min after training and compared with naïve rats (unpaired $t$ test: ${ }^{\star} p=0.0187$ ). Data are expressed as mean \pm s.e.m

locomotor activity suggesting that the significant difference in CFC freezing was not due to mobility alteration (Supplementary Fig. 3a). Similar results were obtained with NOL, as rats injected with WT1-AS exhibited increased memory at $24 \mathrm{~h}$ after training (Fig. 2a). Furthermore WT1-AS injected rats showed short-term memory retention, at $1 \mathrm{~h}$ after training, comparable to SC-ODN injected controls (Fig. 2a), indicating that WT1 in the hippocampus selectively affects long-term memory. The WT1AS or SC-ODN groups did not exhibit any difference in total object exploration time (Supplementary Fig. 3b). These findings, based on two distinct hippocampus-dependent tasks, suggest that WT1, whose expression and DNA binding activity increase following training, decreases memory retention.

To extend the investigation of the role of WT1 on synaptic plasticity and memory to different species, we generated genetically modified mice with forebrain expression of an in-frame internal Wt1 deletion, which produces a truncated WT1 protein that lacks

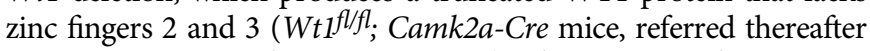
as $W t 1 \Delta$ mice, Supplementary Fig. $4 \mathrm{a}$ ). These protein domains are essential for WT1 DNA and RNA binding activity ${ }^{33}$.

$W t 1 \Delta$ mice were viable, of normal size and weight, and did not show any gross alteration in hippocampal morphology compared with wild type littermates (referred thereafter as Control mice; Supplementary Fig. 4b). The transgenic mice also were similar to Control mice with respect to protein levels in peripheral tissue, as well as in their urine and blood chemistry (metabolic enzyme and electrolyte panel; Supplementary Fig. 4c, d).
Similar to rats in which WT1 was knocked down in the hippocampus, Wt1s mice compared with Control mice showed enhanced memory retention $24 \mathrm{~h}$ as well as 30 days after CFC training (Fig. 2b). They also showed enhancement in NOL retention $24 \mathrm{~h}$, but not $1 \mathrm{~h}$ following training (Fig. 2b). The open field activity, pain response and total object exploration time of $W t 1 \Delta$ mice were similar to those of Control mice (Supplementary Fig. 5a-c), indicating that the effect of the genotype on NOL and CFC were not due to changes in locomotion, pain sensitivity, or exploration. In contrast, when tested in the elevated plus maze, a paradigm used to measure anxiety-like behavior, Wt1 $\Delta$ mice spent significantly more time in the closed arm and made a significant lower number of entries in the open arm (Supplementary Fig. 5d), compared with Control mice, suggesting that forebrain deletion of WT1 may affect also anxiety behavior regulation.

Collectively these results indicate that the expression and functional activation of the transcriptional repressor WT1 is increased in the hippocampus by learning and that WT1 acts to suppress memory.

To test WT1 function as a memory suppressor, we overexpressed wild type WT1 using either an AAV or HSV virus injected into the dorsal hippocampus of rats (WT1-AAV or WT1-HSV; Fig. 2c, d respectively). AAV-GFP or HSV-GFP viruses were used as controls (CTR-AAV or CTR-HSV). Rats bilaterally injected into the hippocampus with either viruses were trained in CFC either 4 weeks (AAV) or 3 days (HSV) after infection, times that correspond to the respective peaks of viral 

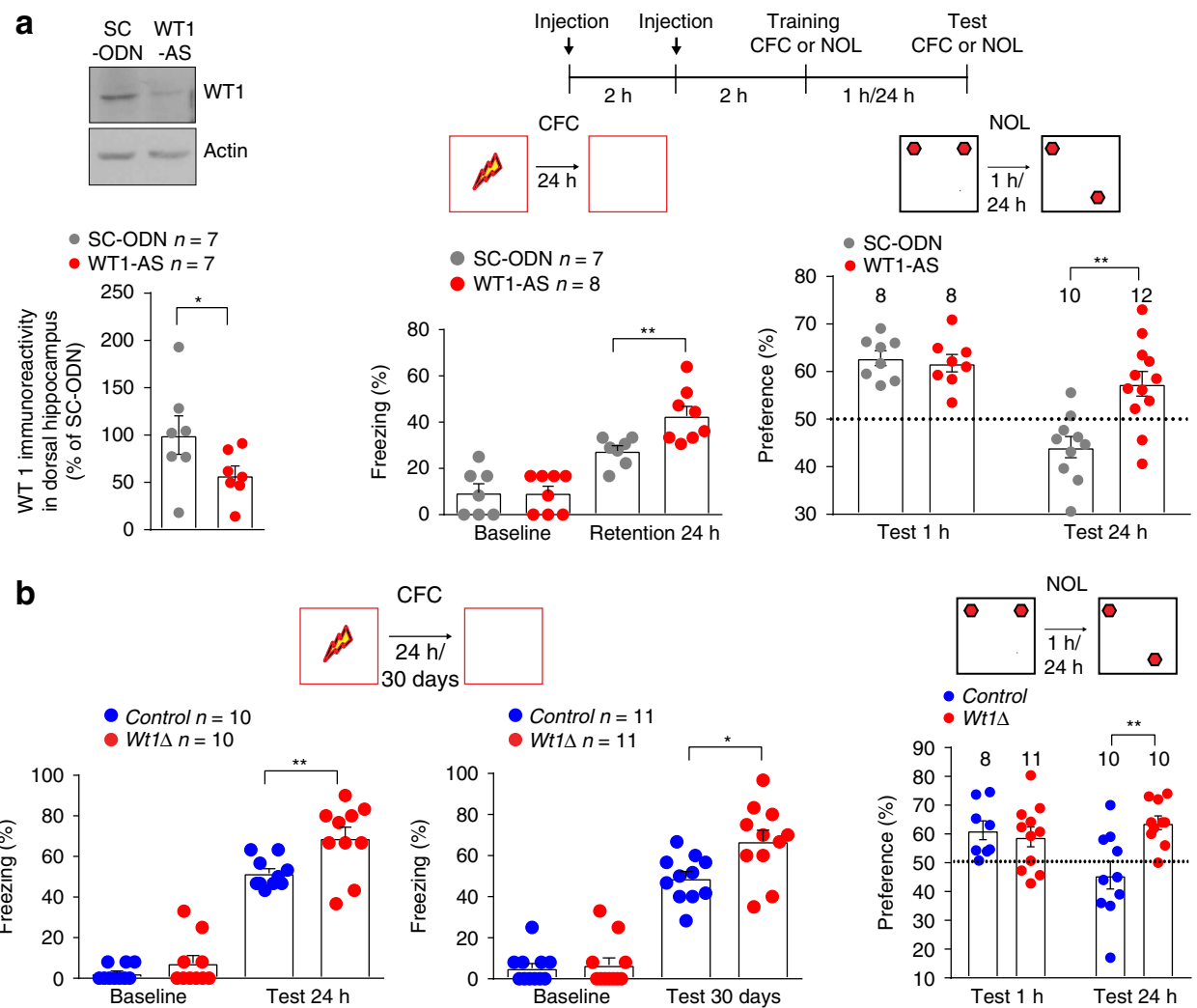

CFC
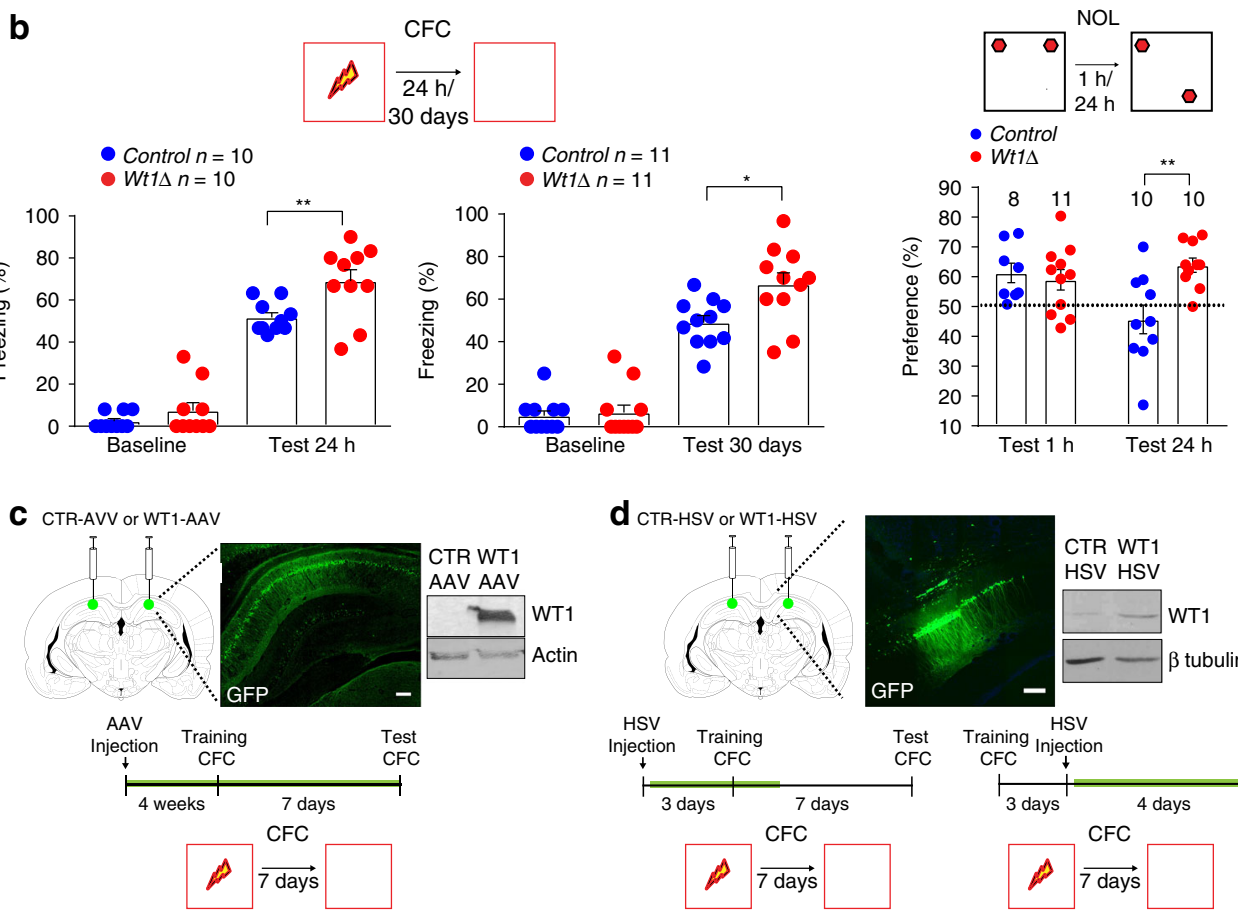

CTR WT1 HSV HSV
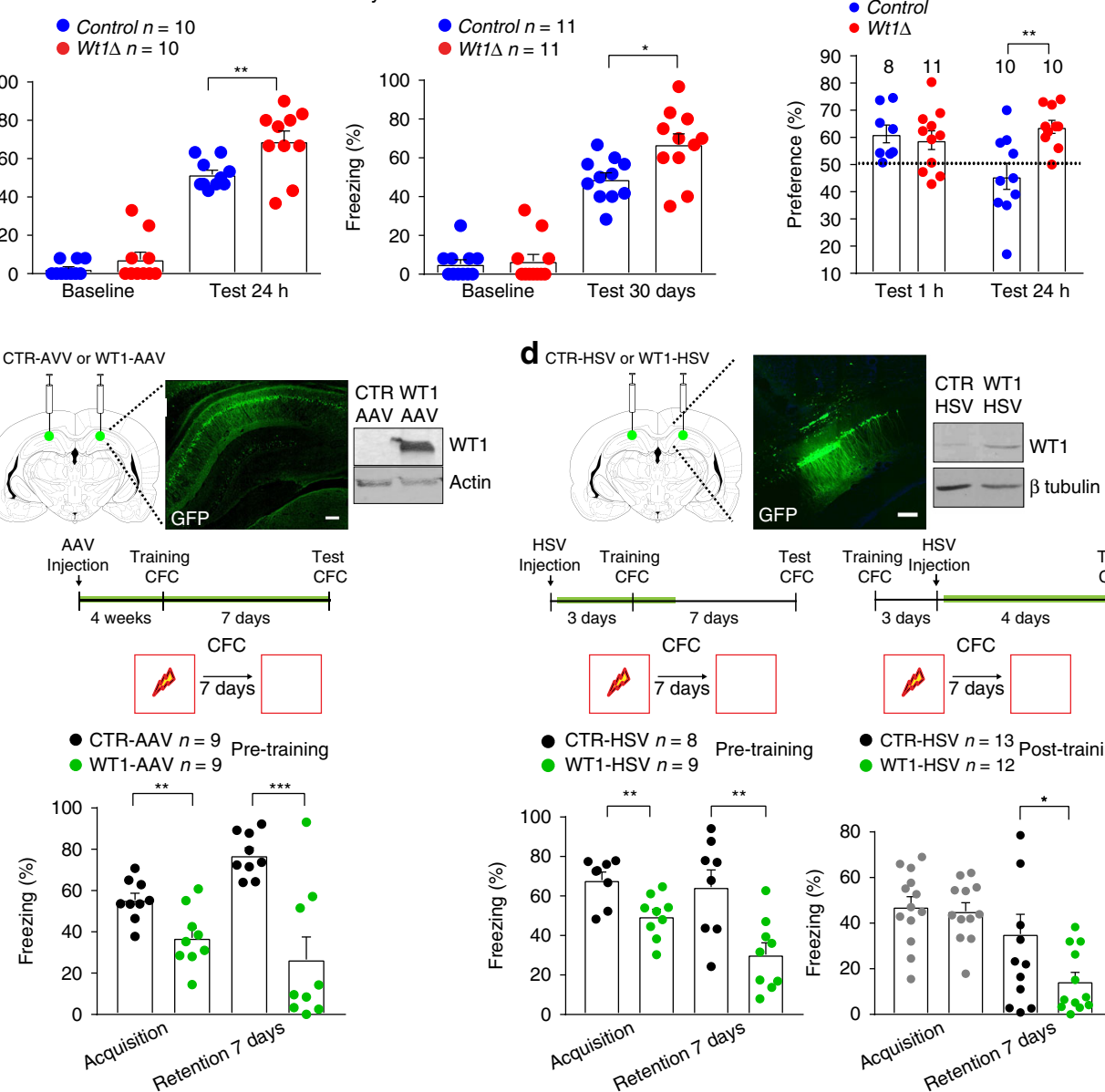

CTR-HSV $n=8 \quad$ Pre-training
WT1-HSV $n=9$

HSV
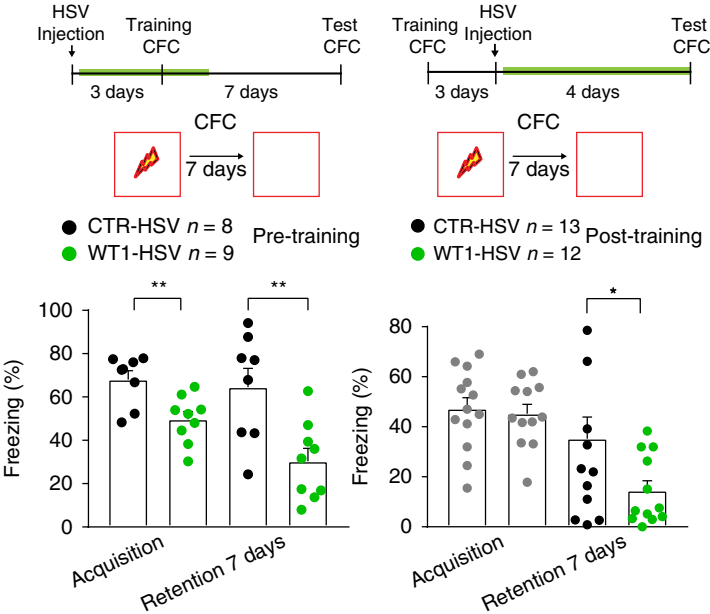

- CTR-HSV $n=13$ Post-training

WT1-HSV $n=12$

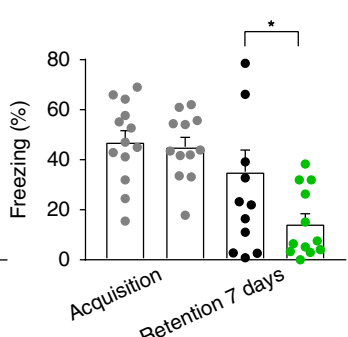

expression for the two viruses. As shown in Fig. 2c, d (pretraining) both WT1-AAV- and WT1-HSV-injected rats had a significantly decreased memory retention 7 days after CFC training. These data indicate that WT1 overexpression is sufficient for reducing memory retention. Notably, because overexpression of WT1 significantly reduced the acquisition of the task (Fig. 2c, d), we tested the effect of viral injection following training (post-training). As shown in Fig. 2d (posttraining), WT1-HSV compared with control virus decreased memory retention tested 7 days after training. Overall our data indicate that overexpression of WT1 is associated with decreased memory retention of an aversive memory.

WT1 controls excitability of hippocampal CA1 neurons. Immunohistochemical staining of rat and mouse hippocampus obtained from naïve animals revealed that WT1 is predominantly localized within the nuclei of pyramidal neurons with a weaker immunoreactivity in the proximal apical dendrites. WT1 immunoreactivity was not detected in astrocytes marked by glial fibrillary associated protein (GFAP-positive) (Fig. 3a-c). 
Fig. 2 WT1 represses long-term memory consolidation. a Left: Change in WT1 expression after double injection ( 2 nmoles/each, $2 \mathrm{~h}$ apart) of WT1-AS into CA1 (paired $t$ test: ${ }^{\star} p=0.0362 ; t=2.687, \mathrm{df}=6$ ). Right: Scheme of behavioral experiments in WT1 knockdown rats. WT1-AS-injected rats increased freezing time $24 \mathrm{~h}$ after training in CFC (unpaired $t$ test ${ }^{\star \star} p=0.0086$ ). WT1 acute knockdown did not affect memory retention in NOL $1 \mathrm{~h}$ after training (unpaired $t$ test $p=0.1685, n=8-12$ rats). In contrast, $24 \mathrm{~h}$ after training, WT1-AS-injected rats showed better memory than SC-ODN injected ones (unpaired $t$ test: ${ }^{\star \star} p=0.0011$. Dashed line indicates $50 \%$ preference). b Left: Wt1 $\Delta$ mice showed enhanced freezing $24 \mathrm{~h}$ and 30 days after training in CFC

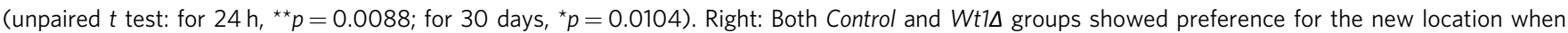
tested $1 \mathrm{~h}$ after training in NOL while only $\mathrm{Wt} 1 \Delta$ mice showed significant preference for the new location $24 \mathrm{~h}$ after training (unpaired $t$ test: ${ }^{\star *} p=0.0033$; $n=8-10$ rats. Dashed line indicates 50\% preference). c Top: Immunostaining and immunoblot showing WT1 overexpression in rats. Bottom: Scheme of the behavioral experiments: green highlight line indicates time window for AAV-induced full expression of WT1. WT1 Overexpression reduced levels of freezing both during acquisition (unpaired $t$ test ${ }^{\star \star} p=0.0061$ ) and 7 days after training (unpaired $t$ test ${ }^{\star \star \star} p=0.0004$ ) compared with CTR-AAV controls. d Top: Immunostaining and immunoblot showing WT1 overexpression via HSV virus in rats. Bottom: Scheme of pre- and post-training behavioral experiments. Green highlight line indicates time window for HSV-induced full expression of WT1. Pre-training: WT1-HSV group showed a significant difference in freezing compared with CTR-HSV group both during acquisition (unpaired $t$ test ${ }^{\star \star} p=0.0042$ ) and 7 days after training (unpaired $t$ test: $\left.{ }^{\star \star} p=0.0049\right)$. Post-training: rats were tested 4 days after HSV injection. WT1-HSV group showed significantly reduced levels of freezing compared with CTR-HSV group (unpaired $t$ test: ${ }^{\star} p=0.0444$ ). Data are expressed as mean \pm s.e.m

Given that the effect of decreasing WT1 expression in the hippocampus enhances memory retention, here we tested whether WT1 knockdown affects hippocampal LTP induction and/or maintenance. Western blot analyses showed that single intrahippocampal injection of WT1-AS significantly decreased WT1 levels in hippocampal slices compared with control slices injected with SC-ODN (Fig. 3d). This WT1 knockdown did not alter basal synaptic transmission (Supplementary Fig. 6a), nor did it affect the induction or maintenance of LTP elicited by StrongHFS (Supplementary Fig. 6b). However, a role for WT1 in synaptic plasticity emerged at synapses activated with a weak high-frequency stimulation (Weak-HFS) protocol, which produced decremental potentiation in control slices but stable LTP in slices from animals injected with WT1-AS (Fig. 3d).

To assess whether WT1 knockdown might enhance LTP indirectly through an effect on interneuron function ${ }^{34}$, we stimulated slices with Weak-HFS in the presence of the $\mathrm{GABA}_{\mathrm{A}^{-}}$ receptor antagonist bicuculline. Under these conditions, hippocampal slices from WT1 knockdown rats still showed enhanced LTP, indicating that WT1 likely regulates synaptic plasticity through direct effects on pyramidal neurons (Fig. 3e).

We therefore hypothesized that WT1 knockdown might enhance LTP by increasing pyramidal cell excitability, since postsynaptic spiking during stimulation facilitates LTP induction $^{35}$. To test this hypothesis, whole-cell recordings were obtained from pyramidal neurons in area CA1 of rat hippocampus. In recordings from WT1-AS injected hippocampi, weak depolarizing currents (20-50 pA) were more likely to evoke action potentials than in neurons of scrambled ODN-injected hippocampi (Fig. 3f) indicating that WT1 knockdown increased excitability. In contrast, in response to relatively strong depolarizing currents (70-100 pA), neurons from WT1-AS slices fired significantly fewer action potentials than those treated with scrambled ODN (mean number of spikes $=2.1 \pm 0.173$ and $1.375 \pm 0.125$, respectively; unpaired $t$-test: ${ }^{\star} p=0.0146 ; t=3.394$, $\mathrm{df}=6)$. No significant differences were observed in the amplitude, frequency or inter-event interval in both spontaneous and mEPSCs (Supplementary Fig. 7a, b).

In agreement with these data in rat hippocampus, slices from $W t 1 \Delta$ mice also showed sustained hippocampal LTP following Weak-HFS, while slices from Control mice produced only transient potentiation (Fig. 3g). When compared with their control littermates, Wt1 $\Delta$ mice showed increased basal Schaffer collateral-CA1 synaptic efficiency with no difference in pairedpulse ratio (Fig. $3 \mathrm{~h}$ ), indicating that WT1 regulates synaptic efficiency through a postsynaptic mechanism.

Collectively, these results suggest that WT1 acts as a synaptic plasticity repressor that dampens the postsynaptic response to a weak stimulus, while preserving the normal dynamic range of the response to super threshold stimuli.

WT1 regulates the computational properties of CA1 cells. The role of the CA1 region in memory processing involves the circuitlevel integration of information arriving from the entorhinal cortex via two major inputs: (1) the direct temporoammonic (TA) pathway, in which entorhinal neurons of the perforant path synapse on distal apical dendrites of CA1 pyramidal neurons, and (2) an indirect input, in which entorhinal activity provides phasedelayed information to proximal apical dendrites in CA1 through a series of three synapses: perforant path $\rightarrow$ dentate gyrus, mossy fibers $\rightarrow$ CA3, and Schaffer collaterals (SC) $\rightarrow$ CA1. The CA1 pyramidal neuron functions as a coincident detector, integrating these temporally segregated streams of information from cortical activity $^{36}$. This coincidence detection function can be studied in hippocampal slices, where the two inputs are activated independently (Fig. 4a; wild type animal) ${ }^{37}$. We reasoned that WT1 levels could regulate the need for convergent activity of both inputs to induce LTP at the Schaffer collateral-CA1 synapse. Depletion of WT1 might allow SC stimulation alone to induce LTP without the added information provided by the TA input (Fig. 4a; WT1 knockdown animal). We tested this hypothesis by stimulating CA1 with theta-burst stimulation (TBS) at both the TA and SC inputs, with SC stimulation phase-delayed relative to TA. In hippocampi from control rats injected with scrambled ODN, induction of LTP required activation of both inputs (Fig. 4b). However, the TA input became dispensable in WT1-depleted hippocampus, so that SC stimulation alone was as effective in producing LTP as dual pathway stimulation (Fig. 4b). Thus, the "normal" level of WT1 imposes a requirement for circuit-level computation in the CA1 neuron, leading to LTP. In contrast, in WT1-depleted hippocampus circuit-level computation no longer is necessary: SC $\rightarrow \mathrm{CA} 1$ activity can induce LTP without confirmatory input from $\mathrm{TA} \rightarrow \mathrm{CA} 1$. Combined with our findings of increased pyramidal cell excitability and altered spike encoding of depolarization in WT1-depleted hippocampus, this result indicates that WT1 activity plays an important role in determining the computational properties of CA1 pyramidal cells.

WT1 downstream targets genes. To identify the target genes of WT1 in hippocampal synaptic plasticity and memory, we compared mRNA-seq profiles of Wt1 $\Delta$ and Control mice. We identified 193 differentially expressed transcripts (Table 1 and Supplementary Data 3).

While transcripts encoding for plasticity and memoryrelated immediate early genes, such as the activity-regulated 

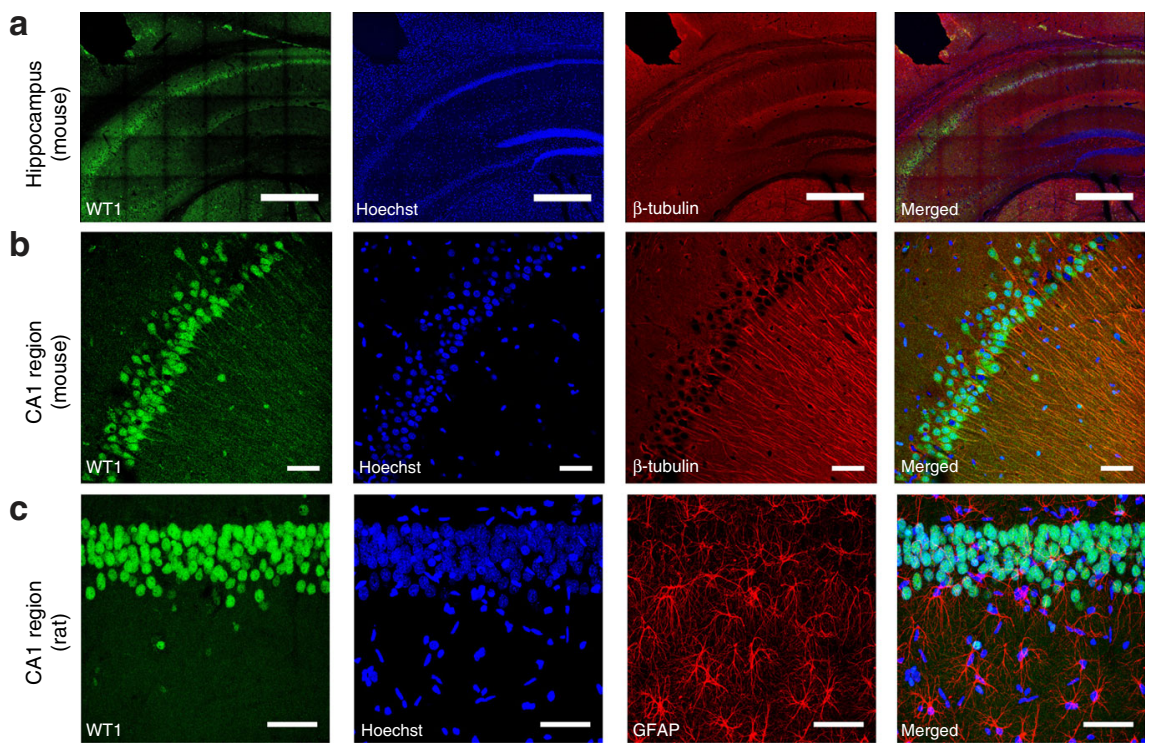

d
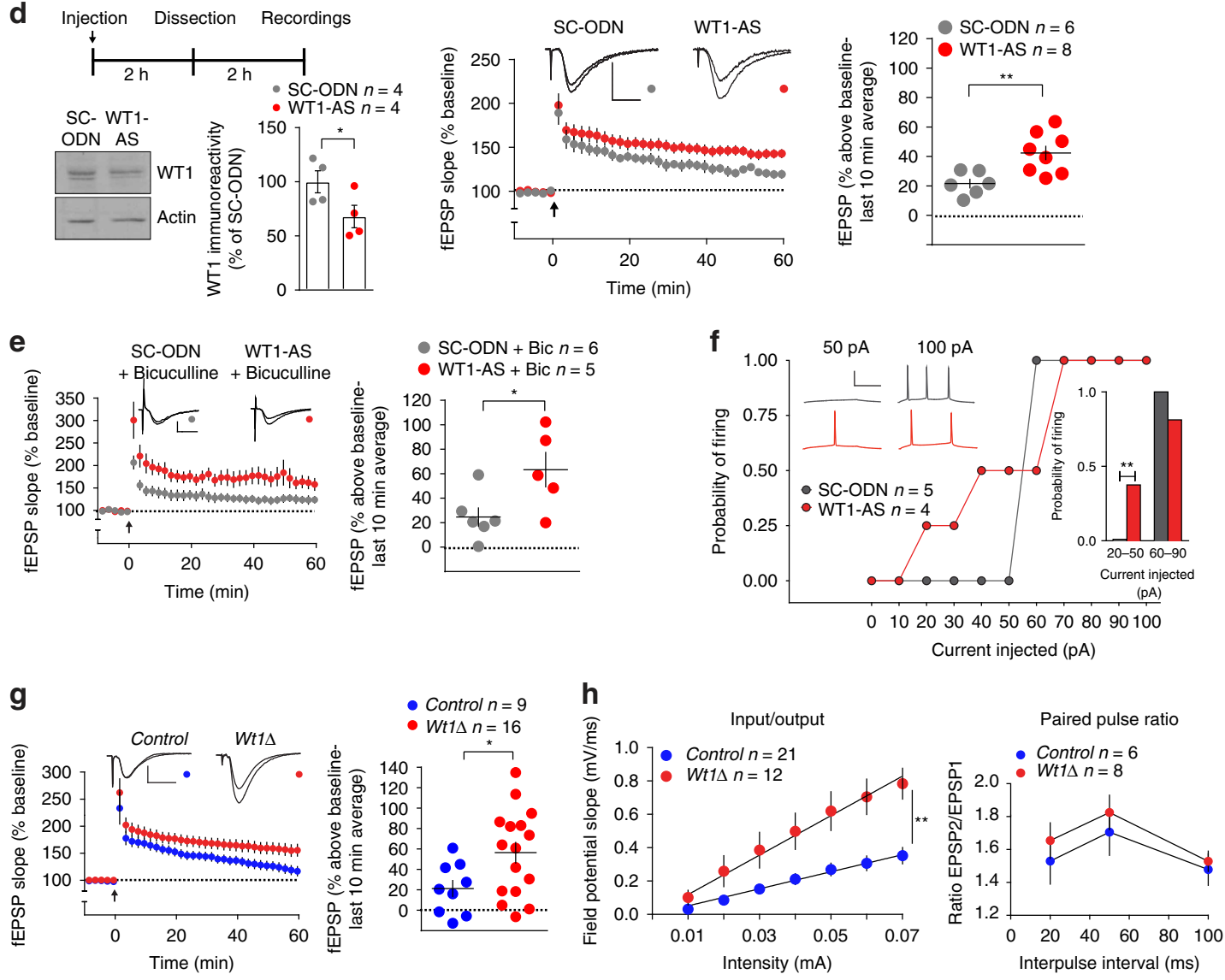

cytoskeletal-associated protein $(A r c)$ and the FBJ osteosarcoma oncogene (Fos), were significantly downregulated, we found that several genes belonging to the retinoic acid signaling pathway were instead upregulated. These include retinol dehydrogenase 5 (Rdh5), cellular retinoic acid binding protein 2 (Crabp2), aldehyde dehydrogenase family 1, subfamily A2 [(Aldh1a2; also known as retinaldehyde dehydrogenase 2 (Raldh2)] (see Table 1 and Supplementary Data 3 for complete list). Interestingly, another upregulated transcript encodes transthyretin (TTR), a protein that is involved with transport of retinol in the plasma and which plays an important role in neuroprotection ${ }^{38}$ as well as memory consolidation and neurogenesis in the hippocampus ${ }^{39,40}$. Furthermore, TTR has also been shown to upregulate hippocampal expression of insulin-like growth factor receptor I (IGFIR) and its nuclear translocation ${ }^{41}$. Notably we found that Igf2 was ranked sixteenth in our list (and the insulin-like growth factor binding protein 2, known as IGFBP2, was ranked fortieth), as one of the top differentially regulated genes. This is in 
Fig. 3 WT1 effect is mediated by enhanced activity and excitability of CA1 neurons. a In the mouse hippocampus WT1 localizes predominantly within the cell bodies layer. Scale bar $=500 \mu \mathrm{m}$. $\mathbf{b}$ Immunostaining of the mouse CA1 region shows WT1 expression mainly in cell bodies but also in proximal dendrites. Scale bar $=50 \mu \mathrm{m}$. $\mathbf{c}$ In the rat CA1 region WT1 is expressed in pyramidal neurons and not in GFAP positive astrocytes. Scale bar $=50 \mu \mathrm{m}$. d Scheme for the electrophysiology experiments. Reduction in WT1 expression after a single intrahippocampal injection of WT1-AS (paired $t$ test: ${ }^{\star} p=$ 0.0202). A weak stimulus (delivered at arrow) induced LTP in WT1-AS group (two-way ANOVA RM: $F_{(1,12)}=10.58,{ }^{\star \star} p=0.0069$ ). Calibrations: $0.5 \mathrm{mV} /$ 10 ms. e, Bicuculline $(10 \mu M)$ did not block WT1-AS-mediated LTP enhancement (two-way ANOVA RM: $F_{(1,9)}=6.039,{ }^{\star} p=0.0363$ ). Calibrations: 0.5 $\mathrm{mV} / 10 \mathrm{~ms}$. $\mathbf{f}$ Whole-cell patch recordings in rats CA1 pyramidal neurons. Right inset: probability of evoking at least one spike in response to a weak (20-50 pA) or a stronger (60-90 pA) current step in WT1-depleted or control groups (two-tailed Chi-square test, $\left.{ }^{\star \star} p=0.0041\right)$. Resting membrane potential and input resistance measured $-63.75 \pm 3.15 \mathrm{mV}$ and $105.8 \pm 21.76 \mathrm{M} \Omega$ in the WT1-AS group, and $-60.80 \pm 2.85 \mathrm{mV}$ and $109.3 \pm 20.04 \mathrm{M} \Omega$ in the SC-ODN group. Left inset: representative traces in cells from WT1-AS or SC-ODN. Calibration: $50 \mathrm{mV} / 100$ ms. $\mathbf{g}$ Upon weak stimulus (delivered at arrow) LTP was induced in Wt1 $\Delta$ mice but not in control group (two-way ANOVA RM: $F_{(1,23)}=5.125,{ }^{\star} p=0.0333$ ). Calibrations: $0.5 \mathrm{mV} / 10 \mathrm{~ms}$. h Wt1 mice showed increased basal synaptic efficiency (left panel: input/output; linear regression unpaired $t$ test, ${ }^{\star \star} p=0.0077$ ) but did not affect paired-pulse ratio (right panel; two-way ANOVA RM, $p=0.0878$ ). Representative fEPSPs graphs show traces recorded during baseline and 60 min post-HFS. Dot blot graphs display final $10 \mathrm{~min}$ of fEPSP slope. Data are expressed as mean \pm s.e.m
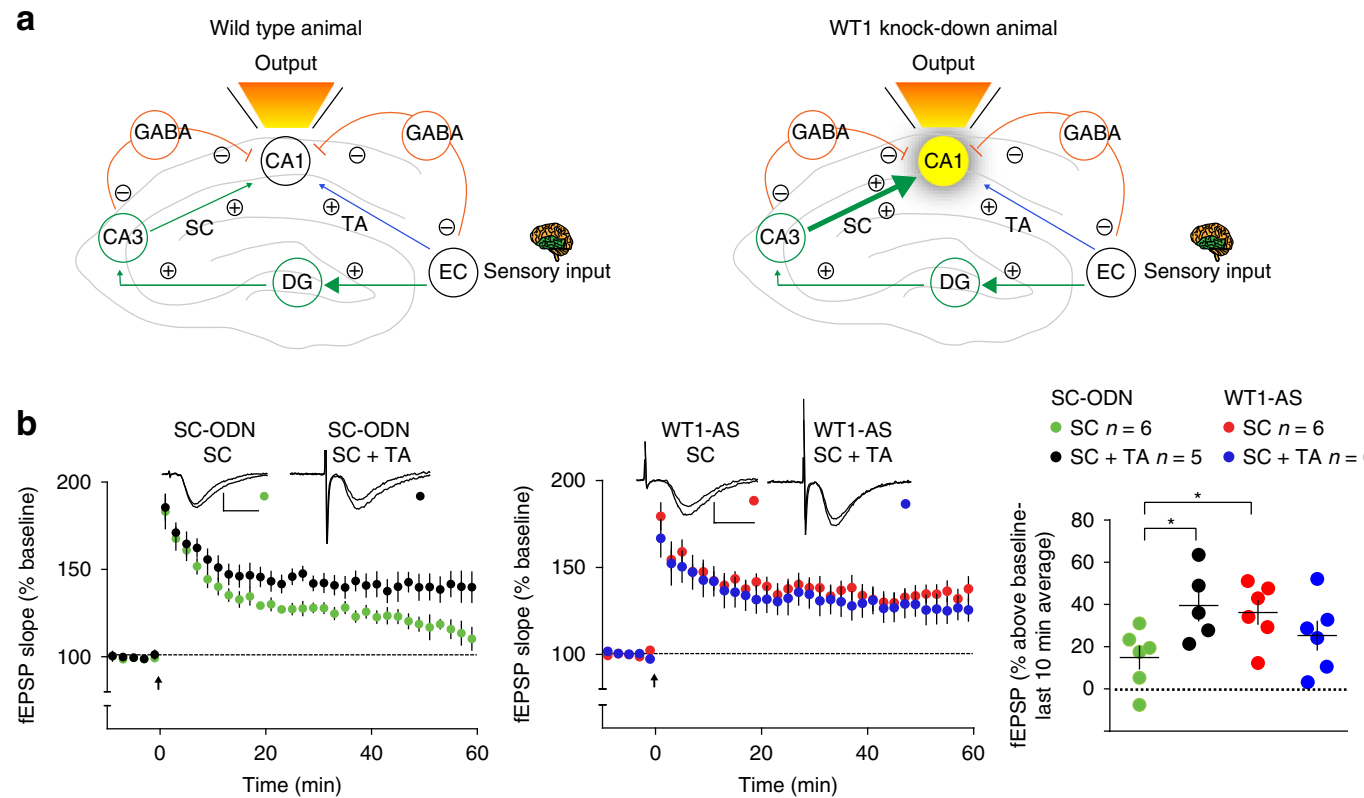

Fig. 4 Circuit mechanism of WT1 action. a Scheme of WT1 depletion effect on corticohippocampal input to CA1. Left panel (wild type animal): normally, activation of both the direct temporoammonic pathway (blue) and the trisynaptic pathway (green) are required for LTP induction at the Schaffer collateral $(\mathrm{SC}) \rightarrow$ CA1 synapse. Right panel (WT1 knock-down animal): in WT1-depleted hippocampus, enhanced basal efficiency of SC $\rightarrow$ CA1 signaling and/or CA1 excitability enable trisynaptic pathway activity alone to induce LTP. EC = entorhinal cortex; DG = dentate gyrus; TA = temporoammonic pathway. $\mathbf{b}$ Theta burst stimulation (TBS, delivered at arrow) of the SC induced stable LTP in slices from rats injected with SC-ODN only when combined with phase-delayed TBS at the TA pathway (left and right panels). Conversely, in slices from WT1-AS-injected hippocampi, the same TBS of SC alone induced LTP, which did not differ from that induced by dual-pathway TBS (center and right panels). Representative fEPSPs show superimposed traces recorded during baseline and $60 \mathrm{~min}$ post-TBS. Calibrations: $0.5 \mathrm{mV} / 10 \mathrm{~ms}$. Data are expressed as mean fEPSP \pm s.e.m. Statistical analysis by two-way ANOVA RM: SC stimulation comparing SC-ODN vs WT1-AS ODNs: $F_{(1,10)}=6.931,{ }^{\star} p=0.0250$; SC-ODN comparing SC stimulation vs SC + TA: $F_{(1,9)}=7.112,{ }^{\star} p=0.0258$. No significant effect was observed when comparing WT1-AS SC vs WT1-AS SC + TA: $F_{(1,10)}=1.437, p=0.2582$

agreement with previous literature on kidney and cell lines (human fetal kidney and HepG2 cells) reporting that WT1 suppresses the expression of Igf2 $2^{42,43}$.

IGF-2 can mediate WT1 effects on synaptic plasticity. In the brain, IGF-2 is required for long-term memory consolidation in the hippocampus, and it has been shown that administration of recombinant IGF-2 significantly enhances memory as well as $\mathrm{LTP}^{44,45}$.

Using quantitative real time RT-PCR, we confirmed that acute WT1 knockdown using WT1-AS significantly increased IGF-2 mRNA expression in dorsal hippocampus (Fig. 5a). Based on this finding, we examined whether Igf2 mediates the enhanced synaptic plasticity produced by WT1-depletion. In hippocampal slices, application of an IGF2 receptor-blocking antibody significantly inhibited LTP enhancement in WT1-deficient mice and rats (Fig. 5b, c) consistent with the hypothesis that, similarly to the kidney, Igf2 is one of the key downstream targets of the transcriptional repressor WT1. Thus, we conclude that the effects on plasticity observed when WT1 is knocked down or ablated rely on derepression of the Igf2 gene.

WT1 enables memory flexibility. A possible role for WT1 is that it limits memory consolidation and strengthening to promote memory flexibility. If this were true, eliminating WT1 function, which results in enhanced CFC, should reduce the ability of the animals to adapt behavioral responses to a changing environment. Thus, we tested whether WT1 depletion affects extinction of CFC memory, reversal learning, repetitive compulsive behavior, and/or sequential learning. Compared to control littermates, 
Table 1 Top 40 differentially expressed genes whose mRNA expression was regulated in the Wt1 $\Delta$ mice. Numbers indicate the $\log _{2}$-fold change for each gene comparing Wt1 $\Delta$ mice with wild type littermates. For the list of all differentially expressed genes, their gene symbols as well as their extended names see Supplementary Data 3

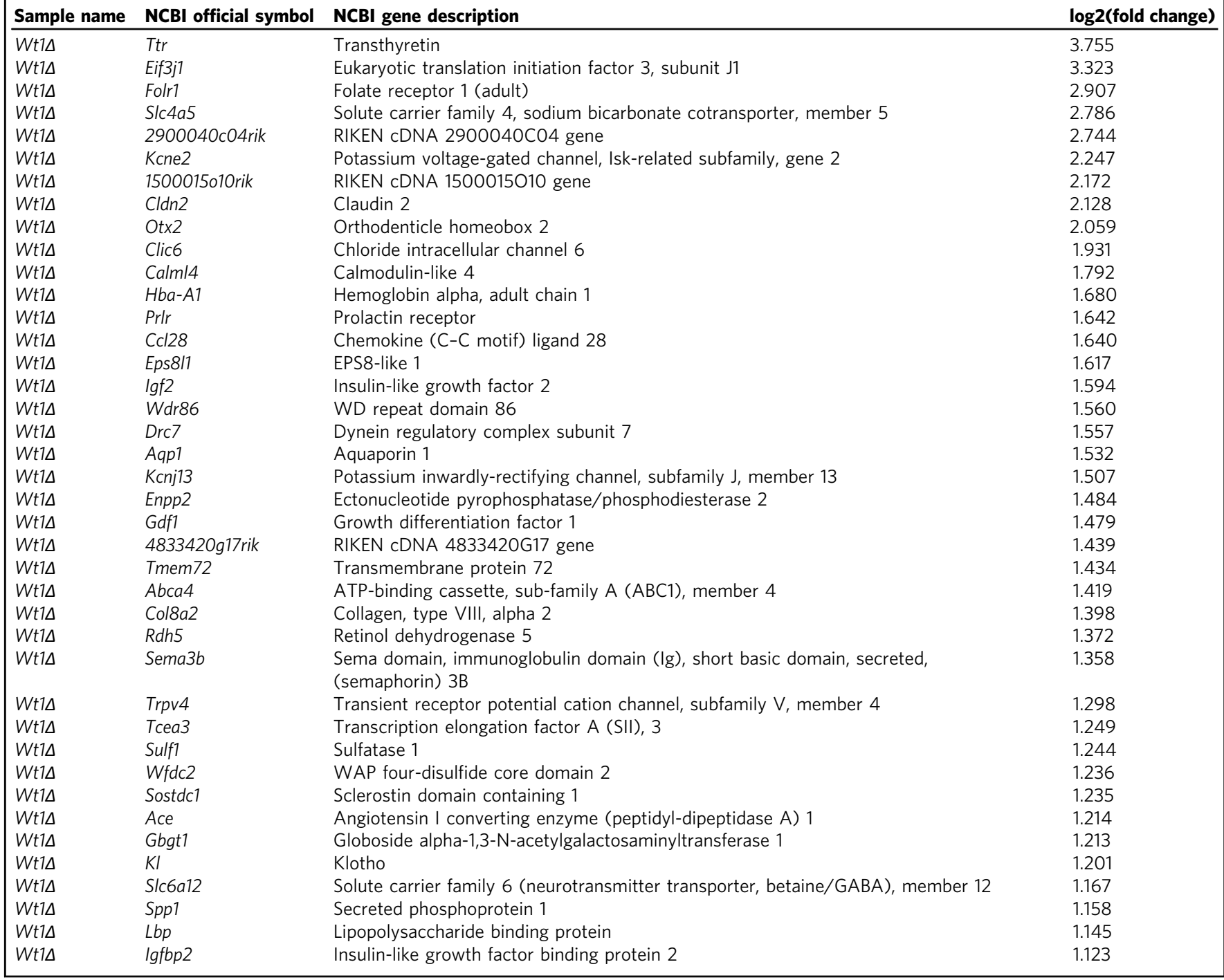

Wt1 mice showed deficient CFC extinction (Fig. 6a), a hippocampal-dependent task by which the animals learn to decrease the conditioned response to fear ${ }^{46}$. Wt1 $\Delta$ mice also showed reduced spontaneous alternation in a Y maze (Fig. 6b), a paradigm widely used to test active retrograde working memory, based on the general trend of mice to explore the least recently visited arm and thus to alternate their visits among the three $\mathrm{arms}^{47}$. Furthermore, when compared to Control, Wt1 $\Delta$ mice showed enhanced memory in the acquisition phase but impairment in the reversal learning phase of the $\mathrm{Y}$ maze (Fig. 6c), indicating that WT1 limits the ability to adapt to previously learned responses. Finally, Wt1 $\Delta$ mice also differ from controls in the marble burying test, a paradigm used to measure repetitive behavior (Fig. 6d).

Together these results indicated that the enhanced memory of Wt1 $\Delta$ mice impacts the abilities of these mice to learn new experiences and flexibly modify their behavior to adapt toward changing environments. These results suggest that sequential learning would be impaired in $W t 1 \Delta$ mice.

To obtain further experimentally testable predictions about possible effects of WT1 on sequential memory, we developed a toy control theory-based model of an information processing and response system. In the model, we postulated that experience activates two parallel pathways: a memorystrengthening pathway that includes transcription factors like CREB and EGR1, and a memory-weakening pathway that includes transcription factors such as WT1. Together the pathways control the activity level of effectors to regulate balance that dictates the level of memory retention. A priori, the total number of effectors could either be in excess of that needed to encode multiple experiences, or they could limit the encoding capacity of the cortico-hippocampal circuit (Fig. 7a). We used the computational toy model to run simulations to study the effect of varying the activity of the memory weakening pathway for a fixed stimulus. The results of the simulations are shown in Supplementary Fig. 8a. The model predicts that if the memory capacity of the cortico-hippocampal circuit is limiting, then over-representation of the first experience could interfere with the ability to acquire subsequent experiences. Alternatively, if effectors were not limiting, then reducing WT1 levels could enhance the ability to memorize both experiences (this is shown schematically in the bar graphs 


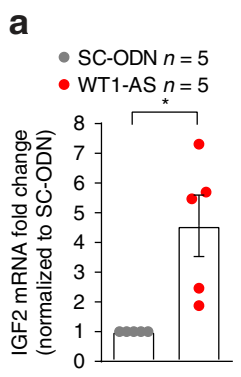

b

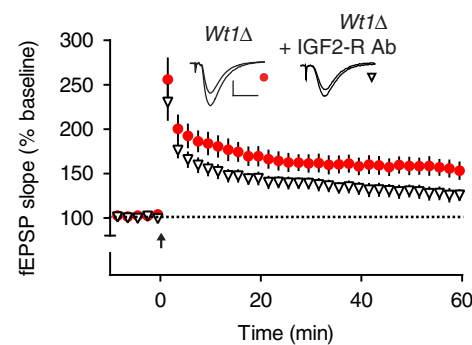

- Control $n=9$

Wt1 $n=16$

$\nabla W t 1 \Delta+$ IGF2-R Ab $n=7$

C
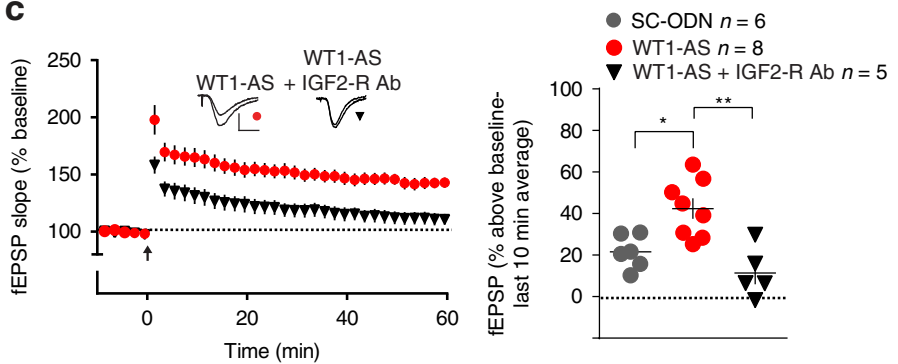

Fig. 5 WT1 effects on hippocampal plasticity are mediated via IGF2. a Quantitative real time PCR showed that WT1 acute knockdown in rats significantly increases IGF2 mRNA expression (unpaired $t$ test ${ }^{\star} p=0.0260$ ). b LTP induced by weak-HFS in Wt1D slices was abolished by bath application of IGF2 receptor antibody (IGF2-R Ab, $5 \mu \mathrm{g} / \mathrm{ml}$ ). Superimposed traces showing representative fEPSPs recorded during baseline and 60 min post-HFS. Calibrations: $0.5 \mathrm{mV} / 10 \mathrm{~ms}$. Summary of the final 10 min of recording showed that LTP in hippocampal slices from Wt1 $\mathrm{mice}$ was significantly reduced by bath

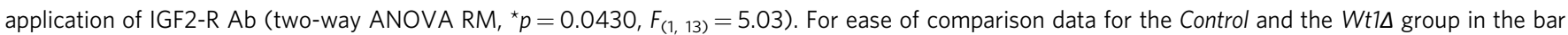
graph are the same as in Fig. 3g. c WT1-AS-mediated LTP enhancement was blocked by bath application of IGF2 receptor antibody (IGF2-R Ab, $5 \mu$ g/ml). Representative fEPSPs show superimposed traces recorded during baseline and 60 min post-HFS. Calibrations: $0.5 \mathrm{mV} / 10 \mathrm{~ms}$. Final $10 \mathrm{~min}$ of recording showed that LTP in WT1-AS injected slices was significantly reduced by IGF2-R Ab (two-way ANOVA RM, ** $p=0.0017 ; F_{(1,11)}=16.85$ ). For ease of

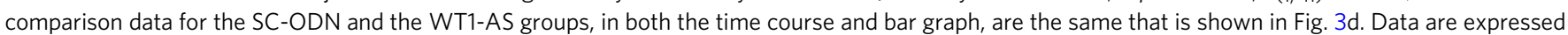
as mean \pm s.e.m

in Fig. $7 \mathrm{a}$ and in the simulation results in Supplementary Fig. 8b; refer to methods section Table 2).

We therefore tested whether WT1 depletion, which enhances memory for one learning, would interfere with new learning in a sequential behavioral paradigm. We first trained mice in NOL, which does not yield long term memory (LTM) at $24 \mathrm{~h}$ after training in Control mice, but does so in Wt1s mice (whereas Control mice shows memory retention at earlier time points, e.g., one hour after training; Fig. 2b). We then exposed the mice to a second learning experience, CFC, which normally does induce LTM (as shown in Fig. 2b). As depicted in Fig. 7b left panel, as expected, $W t 1 \Delta$ mice had significant LTM retention for NOL at $24 \mathrm{~h}$ after training, while Control mice did not. However, when Wt1s mice that first underwent the NOL experience, were exposed one day later to CFC training, they showed significantly reduced LTM for CFC at $24 \mathrm{~h}$ compared with Control mice (Fig. $7 \mathrm{~b}$, right panel), indicating that the first experience learned in the absence of WT1 impacts subsequent learning. In line with these finding, we observed that Wt1 $\Delta$ mice showed significant preference for the new location when tested $48 \mathrm{~h}$ after NOL training (Supplementary Fig. 9a), suggesting a memory interference effect. To determine the duration of this active learning interference, we tested the effect of extending the interval of time between NOL and CFC learning to 10 days. Consistent with previous experiments, $W t 1 \Delta$ mice showed enhanced $24 \mathrm{~h}$ retention for NOL (Fig. 7c, left panel). The 10 days delay between the two sequential experiences resulted in no difference between the two groups in LTM for CFC (Fig. 7c, right panel), indicating that the interference effect is a decaying function of the process induced by the first learning experience and it is temporarily limited. Animals from both groups showed similar exploration time during NOL training for both experiments
(Supplementary Fig. 9b, c). This suggests that strengthening memory by removing WT1 limits behavioral flexibility and that this effect is temporarily restricted.

\section{Discussion}

A better understanding of mechanisms of forgetting is critical for understanding memory storage and persistence. In this study, we identified an important role for the transcriptional repressor WT1 in limiting memory strength by promoting forgetting, which is required for normal flexibility in forming sequential memories. Since WT1, like activator transcription factors including C/EBP, cFos, and Zif $268^{7}$, is induced by LTP and behavioral training, we conclude that the cascade of gene expression that is engaged during learning, and required for long-term memory, requires specific transcriptional repressors in addition to activators. Surprisingly, not many transcription factors have been studied in the context of forgetting and memory flexibility; one example is the transcription activator XBP1, which like WT1 is induced by learning ${ }^{48}$, but acts conversely as a positive regulator of hippocampal long-term memory and flexibility through transcriptional upregulation of brain-derived neurotrophic factor ${ }^{49}$. Given that the role of WT1 is to actively promote forgetting, transcriptional repression via specific DNA binding factors adds to other recently identified mechanisms of active forgetting (processes that counteract memory consolidation and strengthening), which include neurogenesis and Rac1-, dopamine-, and Cdc42mediated AMPA receptor endocytosis ${ }^{3,50}$. Notably, the process of WT1-mediated active forgetting will occur via the function of its target genes, including several members of the retinoic acid signaling pathway (Rdh5, Crabp2, and Ttr), 
a

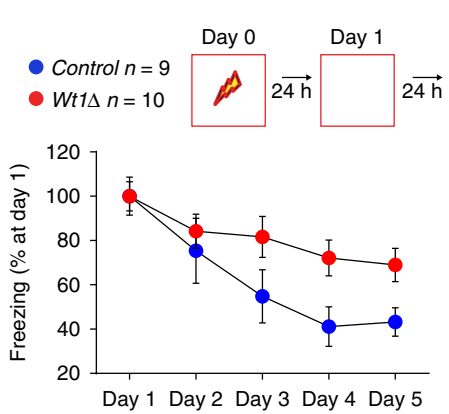

b

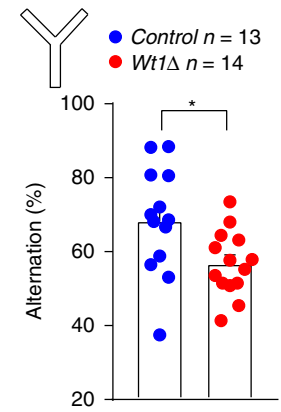

d

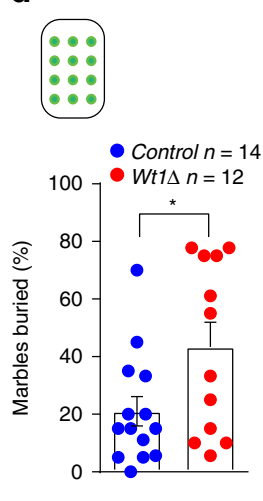

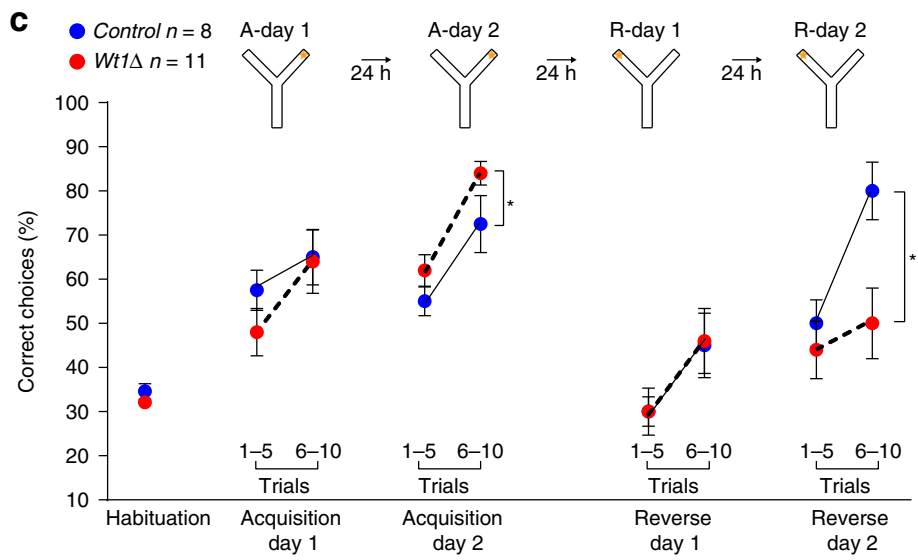

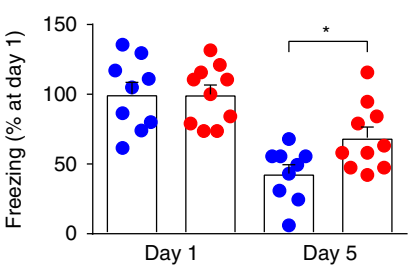

Fig. 6 WT1 controls memory flexibility. a Wt1 1 mice exhibited a lower rate of fear extinction than their control littermates measured at day 5 of extinction;
$\%$ freezing at day 5 was normalized to freezing at day 1 (unpaired $t$ test: ${ }^{*} p=0.0196$ ). b Wt1 mice showed impaired spontaneous alternation (\% alternation) in a $Y$ maze test (unpaired $t$ test: ${ }^{\star} p=0.0178$ ). c $W t 1 \Delta$ mice compared to Control mice performed significant different during the acquisition and reversal phase of the reversal learning task in a $Y$ maze. Data are expressed as \% correct arm entry (baited arm). A-day 1 and A-day 2: acquisition sessions 1-2; R-day 1 and R-day 2: reversal sessions 1-2 (two-way ANOVA RM; ${ }^{\star} p=0.0348 ; F_{(1,17)}=5.263$ for acquisition phase; ${ }^{\star} p=0.0120 ; F_{(1,17)}=$ 7.916 for reversal phase). d, Wt1 1 mice exhibited an increase in repetitive behavior as indicated by the number of marbles buried in the marble burying test (unpaired $t$ test: ${ }^{\star} p=0.0297$ ). Data are expressed as mean \pm s.e.m

the immediate early genes Arc and Fos, as well as Igf2 which our data indicate to signal through the IGF-2 receptor (IGF2R). IGF-2R, also known as cation-independent mannose-6phosphate receptor, binds IGF-2 and other ligands and targets them to lysosomal degradation. Hence, it is possible that lysosomal degradation serves to rebalance and complement the de novo protein synthesis and structural changes induced by learning. Of note, Igf2 is one of the best characterized WT1 target genes ${ }^{42}$, and it has been shown to enhance synaptic plasticity and long-term memory and to prevent memory loss 44,51 , mimicking some of our findings related to WT1ablated animals. However, there are divergences as IGF-2 injected mice show enhanced fear extinction with intact memory flexibility ${ }^{45,52}$, suggesting that induced IGF2 expression can explain only some of the behavioral effects observed in WT1-ablated animals (enhanced plasticity and long-term memory).

One additional observation is that WT1, by regulating its targets, might be involved in the regulation of homeostatic plasticity or synaptic scaling ${ }^{53}$, which is the ability of neurons to respond to periods of reduced or excessive activity by increasing or decreasing, respectively, their synaptic efficiency. Synaptic scaling in excitatory neurons occurs through enhancement, or decrease of, AMPA receptor-mediated transmission, which is in turn regulated by several molecular players ${ }^{53}$. In this regard, active forgetting has also been linked to cytoskeleton targeting mechanisms of synaptic remodeling ${ }^{11,54,55}$ and AMPA receptor recycling 50,56 . The regulation mechanisms underlying homeostatic plasticity and AMPA receptor recycling are still only partially known, but they include some of the WT1 target genes, such as $A r c^{57}$, retinoic acid ${ }^{58}$, and IGF- $2^{44}$. Notably, both retinoic acid and IGF-2 bind to the IGF-2 receptor, which regulates endocytosis and endosomal trafficking, in addition to lysosomal degradation ${ }^{59}$. We suggest that this regulation may influence AMPA receptor trafficking and surface expression, and therefore contribute to synaptic scaling as well as memory-related plasticity. Our data, combined with what is known from the literature, indicate that there are likely to be multiple downstream molecular mechanisms underlying the effects of WT1. Further studies will be needed to determine which mechanisms are operative in a particular context.

Our finding that the expression of nonfunctional WT1 impairs subsequent learning after a first learning experience suggests anterograde interference due to aberrantly strong representation of the first learning experience. The nature of the first task appears important for the time window during which the memory interference effect occurs, as in the sequential learning protocol we found that Wt1 $1 \Delta$ mice still showed preference for the new location $48 \mathrm{~h}$ after training (see Supplementary Fig. 9a), time point at which CFC was performed (see Fig. 7b). However, when the two tests were separated by 10 days, Wt1 $1 \Delta$ animals performed similarly to Control mice in CFC. Different groups have been providing compelling evidence that strong LTP induced by learning can limit the ability to induce further LTP, a phenomenon known as occlusion ${ }^{60-62}$. LTP-occlusion has been shown to impair subsequent learning 
a

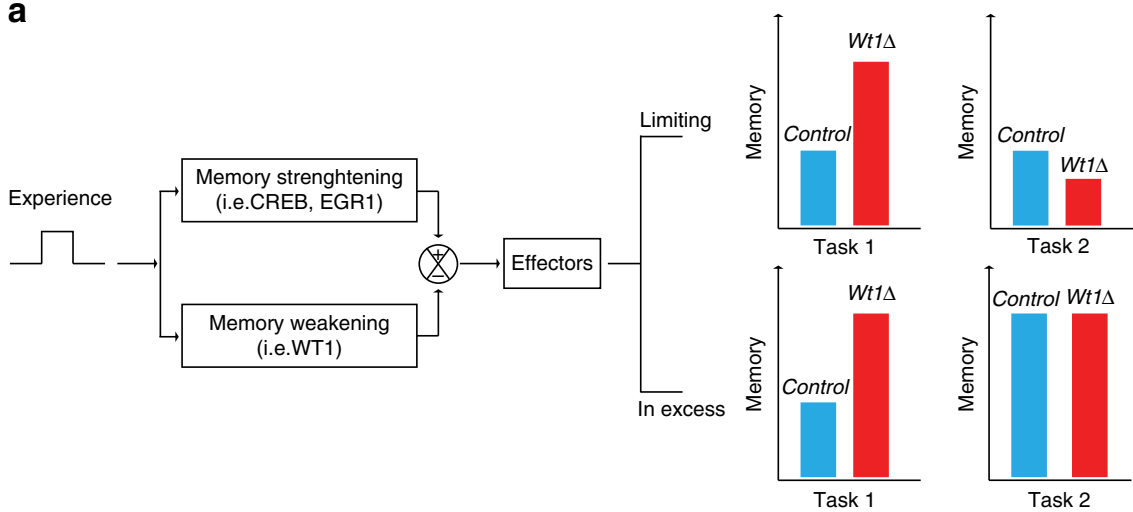

b
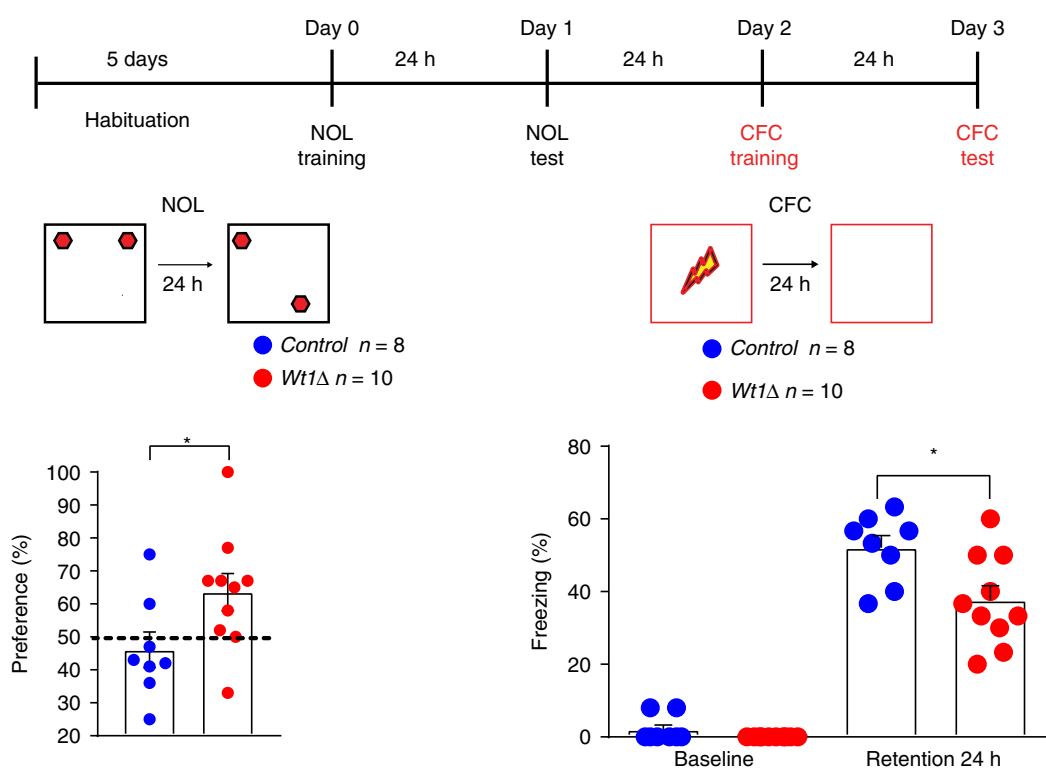

c
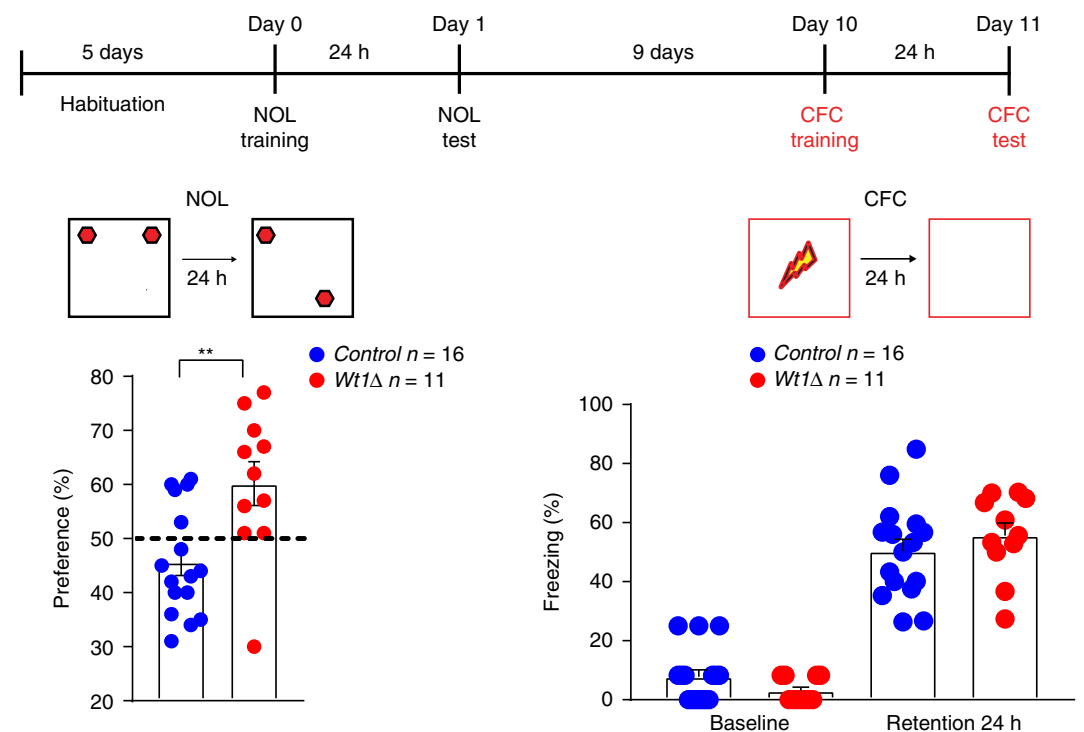

(leading to memory interference) in the hippocampus as well as motor cortex ${ }^{61-63}$. Furthermore, it has been suggested that memory interference is caused by competition for neural resources and that it can persist for hours or days before the capacity of the neurons to undergo LTP is again restored ${ }^{64}$. Based on our data that Wt1 $\Delta$ mice showed enhanced LTP and anterograde memory interference, we cannot rule out the

possibility that a similar mechanism of LTP-occlusion plays a role in the effect observed here. Further investigation is needed to address this question.

The mechanisms that counteract memory strengthening and consolidation are critical for normal memory formation. In fact, these mechanisms, by preventing over-consolidation of memories, allow learning flexibility that supports the ability of the 
Fig. 7 Consequences of WT1 $\Delta$-mediated impaired memory flexibility. a Proposed mechanism of WT1's effect on memory regulation. An initial experience such as Task $1(\mathrm{NOL})$ activates both pro-memory strengthening and pro-memory weakening pathways. When the memory weakening pathways are inhibited by depletion of WT1, there is prolonged memory for Task 1 . Retention of Task 1 memory may or may not interfere with the ability to remember a Task 2 (CFC) based on the availability of effectors (limiting vs in excess). b Schematic representation for short-interval sequential training in mice (top panel). Wt1 $\Delta$ mice showed increased time spent exploring the new location when first trained in NOL and tested $24 \mathrm{~h}$ after training (left panel, unpaired $t$ test: ${ }^{\star} p=0.0422$. Dashed line indicates $50 \%$ preference). In the next day after being tested in NOL mice were trained on CFC and Wt1 $\Delta$ mice spent significantly less time freezing than Control littermates when tested $24 \mathrm{~h}$ after training (right panel, unpaired $t$ test: ${ }^{*} p=0.0161$ ). c Schematic representation for long-interval sequential training (top panel). Wt1 1 mice showed increased time spent exploring the new location when first trained in NOL and tested $24 \mathrm{~h}$ after training (left panel, unpaired $t$ test: ${ }^{\star \star} p=0.0036$. Dashed line indicates $50 \%$ preference). Nine days after being tested in NOL, Wt $1 \Delta$ mice were trained on CFC and compared with control group, they spent comparable amount of time freezing when tested $24 \mathrm{~h}$ after training (right panel, unpaired $t$ test: $p=0.3816)$. Data are expressed as mean \pm s.e.m

\begin{tabular}{|c|c|c|c|}
\hline Parameter & Description & Value & Comments \\
\hline$\tau_{1}$ & $\begin{array}{l}\text { Memory-strengthening signaling time } \\
\text { constant }\end{array}$ & $0.5 \mathrm{~h}$ & $\begin{array}{l}\text { Should be faster than memory-weakening; not } \\
\text { affected by WT1 knockdown }\end{array}$ \\
\hline$\tau_{2}$ & $\begin{array}{l}\text { Memory-weakening signaling time } \\
\text { constant }\end{array}$ & 36 h control; 144 h WT1 knockdown & $\begin{array}{l}\text { Slower than memory-strengthening, prolonged by } \\
\text { WT1 knockdown }\end{array}$ \\
\hline$K_{1}, K_{2}$ & Steady-state gains & 3 control; 7.2 WT1 knockdown & N/A \\
\hline
\end{tabular}

organism to adapt to changing conditions. Particularly important for pathologies in the area of trauma and anxiety was the observation that WT1-depleted mice trained in CFC show decreased extinction and an increased anxiety response, as measured by elevated plus maze. These behaviors are typical of anxiety disorders including PTSD, in which it is well known that memories of the aversive experience and traumas have been overconsolidated $^{65}$. As WT1 ablation in the hippocampus does not affect short-term memory, we suggest that the role of WT1 in forgetting is either to counteract memory consolidation or to impair retrieval, and further studies will be needed to understand this issue.

Accurate consolidation of long-term memories in the corticohippocampal circuit relies on coordinated activity in two major inputs, both originating in the entorhinal cortex but activating hippocampal CA1 neurons either directly, or through the trisynaptic pathway. At the level of CA1 neuron, a nonlinear response to synaptic input might underlie its capacity to function as a coincidence detector that appropriately processes the coherent effects of activity in both pathways ${ }^{66}$. We reported here that depletion of WT1 from the CA1 pyramidal neurons leads to a significant increase in excitability (Fig. $3 \mathrm{f}$ and h), to LTP enhancement (Fig. 3d and g), and to alteration of the intrahippocampal circuit response (Fig. 4). Depletion of WT1 interfered with the ability of CA1 neurons to perform this circuit level computation, as dual input to CA1 neurons was no longer needed to produce LTP.

Lastly, we speculate that the identification of WT1 as a new transcriptional regulator of memory persistence and memory flexibility may have potential implications for the treatment of those neurological conditions where memory is inflexible and excessively resistant to disruption, such as PTSD and OCD.

\section{Methods}

Replication, blinding, and statistical analysis. Experiments were run at least three separate times. For the $W t 1 \Delta$-mRNA seq experiment, the results represent two different biological replicates. For behavior experiments the results are obtained from pulling together multiple animals from at least two different cohorts. Details of replicates are provided in each experiment. No statistical methods were used to predetermine sample sizes, but our sample sizes are similar to those reported in previous publications.
}

For all the electrophysiology and behavior experiments, the experimentalists were blind to the mice genotype or to the type of oligonucleotide or AAV/HSV virus treatment during the entire data gathering process. Only after the data were pooled and analyzed was the coding for the different groups revealed.

Unless otherwise stated, data are represented as mean \pm s.e.m. All the statistical analyses were run in GraphPad Prism 7.02.

Research animals. All animal experiments were performed according to ethical regulations and protocols approved by the internal Animal Care and Use Committee at Icahn School of Medicine at Mount Sinai.

Transcription factor activation arrays. Nuclear and cytosolic extracts were isolated according to standard procedures using low speed centrifugation. All buffers contained protease and phosphatase inhibitors. Tissue was lysed using a motorized Potter-Elvehjem homogenizer ( 10 strokes) in Buffer A (20 mM HEPES ( $\mathrm{pH} 7.4)$, $40 \mathrm{mM} \mathrm{NaCl}, 3 \mathrm{mM} \mathrm{MgCl}, 0.5 \% \mathrm{NP}-40,10 \%$ glycerol, $1 \mathrm{mM} \mathrm{DTT}$ ). Homogenized tissue was left for $10 \mathrm{~min}$ on ice, and lysates were spun at $500 \mathrm{~g}$ for $10 \mathrm{~min}$ at $4{ }^{\circ} \mathrm{C}$ to pellet nuclei. Nuclei were washed gently in Buffer B (20 mM HEPES (pH 7.4), $40 \mathrm{mM} \mathrm{NaCl}, 3 \mathrm{mM} \mathrm{MgCl}, 0.32 \mathrm{M}$ Sucrose, $1 \mathrm{mM} \mathrm{DTT}$ ) and spun at $500 \mathrm{~g}$ for $10 \mathrm{~min}$ at $4{ }^{\circ} \mathrm{C}$. Nuclei were then resuspended using equal volumes of Buffer $\mathrm{C}$ (20 mM HEPES (pH 7.4), $40 \mathrm{mM} \mathrm{NaCl}, 1.5 \mathrm{mM} \mathrm{MgCl}, 25 \%$ glycerol, $1 \mathrm{mM}$ DTT) and of Buffer D (20 mM HEPES (pH 7.4), $800 \mathrm{mM} \mathrm{KCl}, 1.5 \mathrm{mM} \mathrm{MgCl}, 1 \% \mathrm{NP}-40$, $25 \%$ Glycerol, $0.5 \mathrm{mM}$ EGTA, $1 \mathrm{mM}$ DTT). Samples were then rotated at $4{ }^{\circ} \mathrm{C}$ for $30 \mathrm{~min}$ to extract nuclear proteins and the resulting lysates were then spun at $13,000 \mathrm{RPM}$ for $20 \mathrm{~min}$ at $4^{\circ} \mathrm{C}$.

The supernatant containing nuclear proteins was used to study transcription factors activation using the Panomics Combo Protein-DNA Array (Affymetrix, MA1215, now sold by Isogen Life Science). Each array membrane is spotted with 345 oligonucleotides that correspond to consensus binding sites for different transcription factors. The location on the array of each consensus binding site, as well as the complete protocol are available in the manufacturer's website http:// www.isogen-lifescience.com/tf-protein-dna-array). Five micrograms of nuclear extract was incubated with the biotinylated probe mix from the array kit for $30 \mathrm{~min}$ at $15^{\circ} \mathrm{C}$. These probes are also transcription factor consensus binding sites that are complementary to the oligonucleotides spotted on the array. Probes that bound to transcription factors in the nuclear extract were purified by spin column separation, and bound probes were further purified from the transcription factors according to the manufacturer's instructions. The purified probes were boiled for $3 \mathrm{~min}$ and hybridized overnight at $42^{\circ} \mathrm{C}$ to the array containing 345 oligonucleotide transcription factor consensus binding sites. The array was then washed, blocked, incubated with Streptavidin-HRP, and visualized by enhanced chemiluminescence The blot was scanned and spot intensities were quantified using Image J.

For each condition (control and stimulated $30 \mathrm{~min}$ ), ten CA1 regions were dissected from hippocampal slices obtained from at least three different animals and were pooled together in order to obtained sufficient nuclear extracts $(5-10 \mu \mathrm{g})$. We compared extracts from unstimulated (control) slices with extracts from slices that were stimulated with Strong-HFS (see field recordings section within electrophysiology methods) and collected 30 min after stimulation. 
Gel shift assay-EMSA. DNA probes were prepared by annealing complementary single-stranded oligonucleotides with 5'GATC overhangs (Genosys Biotechnologies, Inc.) and labeled by filling in with $[\alpha-32 \mathrm{P}] \mathrm{dGTP}$ and $[\alpha-32 \mathrm{P}] \mathrm{dCTP}$ using Klenow enzyme. For the CFC experiment, DNA probes were prepared using the LightShift Chemiluminescent EMSA Kit (Thermo Scientific) where complementary single-stranded transcription factor binding consensus sequence was first biotinylated using the Biotin $3^{\prime}$ End Labeling Kit (Thermo Scientific) and then annealed. In both experiments nuclear extracts were incubated with labeled DNA probes for $30 \mathrm{~min}$ at room temperature $\left(22-24^{\circ} \mathrm{C}\right)$. For the LTP experiment DNAbinding complexes were separated by electrophoresis on a $5 \%$ polyacrylamide-Tris/ glycine-EDTA gel which was dried and exposed to X-ray film. For the CFC experiment protein/DNA complexes were separated using a 6\% DNA retardation gel (Invitrogen) that was electroblotted into a Biodyne B membrane (Thermo Scientific), incubated with Streptavidin-HRP (Thermo Scientific) and visualized by ECL according to the manufacturer's instructions. The consensus sequence used for WT1 was: 5'-AATTCGGGGGCGGGGGCGGGGGCGGGGGAGGGGCGC-3' and its complementary sequence. For the $\mathrm{CFC}$ experiment binding was confirmed using an additional consensus sequence $5^{\prime}$ - TCCTCCTCCTCCTCTCCC-3'.

For the LTP experiments, slices were stimulated using Strong-HFS protocol (see field recordings section within electrophysiology methods); for the CFC experiment, animals were trained using three footshocks protocol $(2 \mathrm{~s}, 0.65 \mathrm{~mA}$, $1 \mathrm{~min}$ apart). The control animals (indicated as "C") remained in the conditioning chamber for the same amount of time as the ones receiving the shock (indicated as "S") but without receiving any footshock.

Real time quantitative RT-PCR. Hippocampal total RNA was extracted with TRIzol (Invitrogen) and $1 \mu \mathrm{g}$ of total RNA was reverse-transcribed using SuperScript III First-Strand Synthesis System (Invitrogen, ThermoScientific, catalog \#18080-051). Real-time PCR was performed using 7500RT PCR System (Applied Biosystems). $1 \mu \mathrm{l}$ of the first-strand cDNA was subjected to PCR amplification using a QuantiTect SYBR Green PCR kit (Qiagen). IGF-II primers (forward: 5'-C CCAGCGAGACTCTGTGCGGA-3'; reverse, 5'-GGAAGTACGGCCTGAGAGG TA-3'); Forty cycles of PCR amplification were performed as follows: denaturation at $95^{\circ} \mathrm{C}$ for $30 \mathrm{~s}$, annealing at $55^{\circ} \mathrm{C}$ for $30 \mathrm{~s}$ and extension for $30 \mathrm{~s}$ at $72{ }^{\circ} \mathrm{C}$. GAPDH (forward, 5'-TGCACCACCAACTGCTTAGC - $3^{\prime}$; reverse, $5^{\prime}$-GGCATGG ACTGTGGTCATGA $-3^{\prime}$ ) was used as internal control. To determine the relative quantification of gene expression the cycle threshold method $\left(C_{\mathrm{T}}\right)$ was used.

Immunohistochemistry. Rats and mice were deeply anesthetized, perfused using $4 \%$ paraformaldehyde and coronal or hippocampal brain sections were obtained using a vibratome (Leica VT 1000 S vibratome; $40 \mu \mathrm{m}$ ) or a cryostat (Leica CM1850; $15 \mu \mathrm{m}$ ). Brain slices were then blocked with $3 \%$ normal goat serum (Vector), $0.3 \%$ Triton X-100 (Sigma-Aldrich), 1\% BSA (Sigma-Aldrich) for $2 \mathrm{~h}$ at room temperature and incubated with the appropriate primary antibody: rabbit monoclonal WT1 (for staining in Fig. 3a Santa Cruz, catalog \#SC-192 (C-19); for staining in Fig. 3b Novus Biological, catalog \#NBP1-40787; for staining in Fig. 3c Abcam, catalog \#ab52933); mouse monoclonal glial fibrillary acidic protein, GFAP (Cell Signaling, catalog \#3670); mouse monoclonal $\beta$-tubulin (Cell Signaling, catalog \#86298). An antibody against green fluorescent protein-GFP (chicken anti-GFP, from Aves Labs Inc., catalog \#GFP-1020) was used to check viral spread in WT1 overexpression experiments using AAV and HSV viruses (Fig. 2c, d). After incubation with primary antibodies, sections were washed and incubated with secondary antibodies complexed to either Alexa Fluor 568 or Alexa Fluor 488 dyes (Invitrogen, ThermoFisher). Please refer to Supplementary Data 4 for complete list of antibodies used for this study. After washing, Hoechst 33342 (Invitrogen) was used to label nuclei. Sections were then mounted and imaged using a confocal microscope (Zeiss LSM 880).

Western blotting. We used either CA1 regions dissected from $400 \mu \mathrm{m}$-thickness hippocampal slices or dorsal hippocampus, homogenized in proportional volumes of ice-cold lysis buffer using a motorized Potter-Elvehjem homogenizer ( $\sim 10$ strokes). The lysis buffer consisted of $50 \mathrm{mM}$ Tris-HCl, $\mathrm{pH} 7.4,100 \mathrm{mM}$ $\mathrm{NaCl}, 1 \mathrm{mM}$ EDTA, $0.5 \%$ sodium deoxycholate, $1 \%$ NP-40, 0.1\% SDS, $0.5 \mathrm{mM}$ PMSF, $1 \mu \mathrm{M}$ mycrocystine, $1 \mu \mathrm{g} / \mathrm{ml}$ benzamidine, $2 \mathrm{mM}$ dichlorodiphenyltrichloroethane (DTT), $1 \mathrm{mM}$ sodium orthovanadate, $2 \mathrm{mM}$ sodium fluoride, $1 \mathrm{mM}$ EGTA; protease inhibitor cocktail (Sigma-Aldrich) and phosphatase inhibitor cocktails 2 and 3 (Sigma-Aldrich) were added according to manufacturer's instructions. Lysates were cleared by centrifugation at 14,000 RPM for $10 \mathrm{~min}$. Protein concentration was determined using Bradford reagent (Biorad). 20-50 $\mu \mathrm{g}$ of total protein was loaded per well, into $10 \%$ SDS-PAGE and transferred to supported nitrocellulose membranes (pore size $0.2 \mu \mathrm{m}$, Biorad), followed by western blotting and chemiluminescence detection. The following antibodies were used: rabbit polyclonal WT1 (custom-made, against a synthetic rat-specific peptide; GeneScript), rabbit monoclonal WT1 (Novus Biological, catalog \#NBP1-40787), mouse monoclonal $\beta$-tubulin (Cell Signaling, catalog \#86298), mouse monoclonal $\beta$-actin (Sigma-Aldrich, catalog \#A4700), mouse monoclonal GAPDH (SigmaAldrich, catalog \#G8795). Please refer to Supplementary Data 4 for complete list of antibodies used for this study. We either use films that were scanned and signal intensity analyzed using either ImageJ or Odyssey. Uncropped blots are provided as Supplementary Fig. 10 .

For both electrophysiology (Fig. 1e, Supplementary Fig. 2a) and behavior (Fig. 1f, g, Supplementary Fig. 2d) experiments time point chosen was 30 min after the delivery of Strong-HFS, LFS or the aversive stimulus (shock). Total protein lysates were collected from CA1 region (electrophysiology) or from dorsal hippocampus (behavior) and processed as described above. For the IA experiment, trained animals were compared with Naive ones. For additional information about the protocol used for LTD and CFC experiments reported in Supplementary Fig. 2, refer to the electrophysiology and behavioral assay sections respectively.

Hippocampal injections of ODNs or HSV/AAV viruses in rats. Animals were anesthetized with a solution containing a mix of ketamine $(100 \mathrm{mg} / \mathrm{kg})$ and xylazine $(20 \mathrm{mg} / \mathrm{kg})(10 \mathrm{mg} / \mathrm{kg}$, intraperitoneal), and a stainless-steel guide cannulae were bilaterally implanted targeting the dorsal hippocampus $(4.0 \mathrm{~mm}$ posterior to bregma, $2.6 \mathrm{~mm}$ lateral from midline, and $2.0 \mathrm{~mm}$ ventral). The rats were returned to their home cages and allowed to recover from surgery for 7-10 days.

For WT1 knock-down experiments all hippocampal injections consisted of $2 \mathrm{nmol}$ in $1 \mu \mathrm{l}$ per side (unless otherwise specified) of either WT1 antisense oligodeoxynucleotide combo (WT1-AS $=1 \mathrm{nmol}$ of WT1 antisense $1+1 \mathrm{nmol}$ of WT1 antisense 2) or scrambled oligodeoxynucleotide combo (SC-ODN $=1 \mathrm{nmol}$ Scrambled $1+1 \mathrm{nmol}$ Scrambled 2) both diluted in phosphate-buffered saline (PBS) at $\mathrm{pH}$ 7.4. The sequences used were the following: WT1 antisense 1: TCG GAACCCATGAGGTGCGG; WT1 antisense 2: TCGGAACCCATGGGGTGC; Scrambled 1: GGTGGTAGAACGCCGTACCG; Scrambled 2: GGTGGTAGAAC GCCGTCC. The scrambled oligonucleotides, which served as a control, were designed to lack homology to any rat sequence in GenBank, and contained the same base composition but in a randomized order. Both antisense and scrambled oligonucleotides were phosphorothioated on the three terminal bases of both $5^{\prime}$ and $3^{\prime}$ ends to increase their stability and were reverse phase purified (GeneLink). For electrophysiology experiments, male Sprague-Dawley rats were used. Animals received a single injection of oligonucleotides $2 \mathrm{~h}$ before being sacrificed, and their brains were dissected (see Fig. 3d for schedule diagram). For electrophysiology experiments one side of the brain was always injected with WT1-AS and the other side of the brain with SC-ODN. For all the behavior experiments either male Sprague-Dawley or Long-Evans rats were used and no differences between the strains were observed. For behavior experiments, animals received two injections of oligodeoxynucleotides $2 \mathrm{~h}$ apart and $2 \mathrm{~h}$ before training (see Fig. 2a for schedule diagram); animals were injected bilaterally with either WT1-AS or SC-ODN.

For overexpression of WT1 via HSV, we used a p1005 based HSV vector coexpressing GFP and WT1-IsoformD (WT1-HSV). In this system, GFP expression is driven by a cytomegalovirus (CMV) promoter, while the WT1-isoformD is driven by the IEF $4 / 5$ promoter. HSV virus expressing GFP alone was used as a control (CTR-HSV). We injected $2 \mu \mathrm{l}$ of HSV vectors in each hemisphere (titer $0.5 \times 10^{\wedge} 9$ infectious unit/ml, Virovek, Hayward, CA) using a 28 -gauge needle that extended $1.5 \mathrm{~mm}$ beyond the tip of the guide cannula and connected via polyethylene tubing to a Hamilton syringe. The infusions of HSV viruses were delivered at a rate of $0.33 \mu \mathrm{min}^{-1}$ using an infusion pump (Harvard Apparatus). The injection needle was left in place for $10 \mathrm{~min}$ after the injection to allow complete diffusion of the solution. Rats were randomized to different treatments.

For WT1 overexpression via AAV, we used AAV8.2-EF1a-WT1-PP2A-GFP (WT1-AAV) and AAV8.2-EF1a-PP2A-GFP (CTR-AAV; both vectors were $1 \times$ $10^{13} \mathrm{vg} / \mathrm{ml}$, Virovek, Hayward, CA) as a control. AAV vectors were injected using a $33 \mathrm{Ga}$ needle attached to a $5 \mu \mathrm{l}$ syringe (Hamilton) $2 \mu \mathrm{l}$ in each hemisphere over a $10 \mathrm{~min}$ period. The needle was left in place for $10 \mathrm{~min}$ to allow for efficient diffusion before removal. Rats were randomized to different treatments.

To verify proper placement of cannula implants or viral injection, rats were deeply anesthetized and perfused $(20 \mathrm{~mL} / \mathrm{min})$ with $4 \%$ of paraformaldehyde (PFA) in PBS, their brains removed and fixed with $10 \%$ (vol) buffered formalin in PBS for $48 \mathrm{~h}$. Brains were then sliced in coronal sections $(40 \mu \mathrm{m})$ and the hippocampus region was examined under a light microscope (for cannulae placement) or confocal microscope (for viral injection). Animals where cannulae were misplaced, viral expression was mostly spread outside of the hippocampus, and serious tissue damage was observed were excluded from the experimental groups.

Generation of functionally deficient WT1 mice. Forebrain-specific deletion of Wt1 was achieved by crossing animals homozygous for the conditional Wt1 knockout allele $\left(W t^{f l f f}\right)^{67}$ with a transgenic line, Camk2a-Cre, (B6.Cg-Tg (Camk2a-cre)T29-1Stl/J; Jackson Lab: http://jaxmice.jax.org/strain/005359.html) in which Cre recombinase expression is driven by the $7.8 \mathrm{~kb}$ promoter of $\mathrm{Ca}^{2+} /$ calmodulin-dependent protein kinase II alpha subunit ${ }^{68}$. Progeny were crossed to obtain $W t t^{f l f f}$; Camk2a-Cre (referred through the paper as Wt1L mice) and littermate control animals (referred through the paper as Control mice). Expression of Cre recombinase resulted in the in-frame deletion of of exons 8 and 9 [see Fig. 1e of Gao et al. ${ }^{67}$ ], and generated a truncated allele encoding a shortened non functional WT1 protein lacking zinc fingers 2 and 3. Expression of the recombined Wt1 allele was detectable in the mouse forebrain (see Fig. 3a of Gao et al. ${ }^{67}$ ), and its detection was performed using the following primers: Primer WT1 Delta Forward 5' GCT AAC ATA TGG GAG ACA TT 3' and Primer WT1 Delta 
Reverse $5^{\prime}$ TGC CTA CCC AAT GCT CAT TG 3'. As reported by others, heterozygous Wt1 mice develop kidney nephropathy and glomerulosclerosis ${ }^{69}$, which we have not observed at any time in the Wt1 $1 \Delta$ mice. To further address this issue, we evaluated proteinuria since loss of kidney function is associated with increased levels of proteins in the urine. Using Chemstrips (Roche), we found that there was no significant difference between proteinuria levels of Wt1A mice compared with their control littermates as indicated by the color of the top strips (Supplementary Fig. 4c). We further confirmed that kidney function was normal and that there was no significant difference in the enzymatic values of $W t 1 \Delta$ mice through a pathology screening of their blood samples performed at the Comparative Pathology Center of Mount Sinai (Supplementary Fig. 4d).

To genotype the animals, we used the following primers for the LoxP allele: Primer LoxP Forward 5' CCT TTT ACT TGG ACC GTT TG $3^{\prime}$ and Primer LoxP Reverse 5' GGG GAG CCT GTT AGG GTA 3'. For the Cre allele we used the following primers: Cre Primer Forward 5' GCG GTC TGG CAG TAA AAA CTA TC $3^{\prime}$ and Cre Primer Reverse 5' GTG AAA CAG CAT TGC TGT CAC TT 3' (as indicated in the genotyping section by Jackson lab at http://jaxmice.jax.org/strain/ 005359.html).

Wt1 1 animals were viable and had a normal life span, normal body weight, normal fertility and a normal growth rate compared with control littermates.

Throughout the study control wild-type littermates are indicated as Control and they comprise the following subgroups: $\mathrm{Wt1}^{+/+}$; Camk2a-Cre positive, $\mathrm{Wt1}^{+/+}$; Camk2a-Cre negative, $W t 1^{f l+}$; Camk2a-Cre negative, $W t 1^{f l / f l}$; Camk2a-Cre negative. These were grouped together for both electrophysiology and behavior experiments, since they were no statistically different between the genotypes.

Hematoxylin and eosin (H\&E) staining. H\&E staining was performed in order to verify if there was any macroscopic abnormality in brain tissue of Control and $W t 1 \Delta$ mice. Animals were deeply anesthetized with a solution containing ketamine + xylazine and perfused transcardially with ice-cold $10 \%$ formalin. The brains were embeded in paraffin and sliced into $2 \mu \mathrm{m}$ thick sections for staining The sections were de-paraffinized in xylene, rehydrated in graded ethanol series, stained with Mayer's Haemalaun (Carl Roth, Karlsruhe, Germany) for 5 min, washed again, and stained with $1 \%$ eosin (Carl Roth, Karlsruhe, Germany for $2 \mathrm{~min}$ ). The sections were washed in water, dehydrated in graded ethanol series, treated with xylene and mounted for imaging (Supplementary Fig. 4b).

Contextual fear conditioning and extinction. Mice or rats were handled for $3 \mathrm{~min}$ per day for 5 days before training. The conditioning chamber consisted of a rectangular Perspex box (VFC-008: $30.5 \times 24.1 \times 21.0 \mathrm{~cm}$, Med Associates) with a metal grid floor (Model ENV-008 Med Associates) through which the footshock was delivered. The experiment was conducted in a sound-attenuated room, with low levels of light and white noise background.

For animals that underwent paired fear conditioning, the training session consisted of $2 \mathrm{~min}$ exploring the context prior to the delivery of either one footshock ( $2 \mathrm{~s}, 0.65 \mathrm{~mA}$ ) or three footshocks $(2 \mathrm{~s}, 0.65 \mathrm{~mA}, 1 \mathrm{~min}$ apart); after that animals remained in the chamber for additional 2 min before returning to their home cages. Control animals were allowed to explore the context for exactly the same time as the shocked groups without receiving any footshock. Rats trained in the unpaired fear conditioning were placed in the context and after $5 \mathrm{~s}$ one footshock $(2 \mathrm{~s}, 0.65 \mathrm{~mA})$ was delivered. Animals stayed in the box for additional $20 \mathrm{~s}$. Control group was exposed to the context for $25 \mathrm{~s}$ without receiving any footshock. Animals from all groups were tested $24 \mathrm{~h}$ after training and 30 days after training (mice only). Test session consisted in placing the animals back into the conditioning chamber for $5 \mathrm{~min}$ in the absence of any footshock and freezing behavior was recorded. For mice memory extinction experiment, $24 \mathrm{~h}$ after CFC training, animals were placed into the conditioning chamber for five consecutive days, $5 \mathrm{~min}$ each day in the absence any footshock and freezing was scored. Sessions were recorded using a digital video camera, and freezing behavior defined as lack of movement besides heart beat and respiration, was scored every $10 \mathrm{~s}$ by trained observers blind to the experimental conditions. The number of scores indicating freezing (reported in Fig. 2a only) were calculated as a percentage of the total number of observations. Ethovision (Noldus Information Technology) was used to measure percentage of freezing time in all experiments involving mice and rat experiments with WT1 overexpression and in those reported in Supplementary Fig. 2b, c.

Inhibitory Avoidance (IA). IA was carried out as described previously ${ }^{44}$. Briefly the IA chamber (Med Associates) consisted of a rectangular Perspex box divided into a safe compartment and a shock compartment. The safe compartment was white and illuminated, whereas the shock compartment was black and dark. The chamber was located in a sound-attenuated, non-illuminated room. Footshocks were delivered though the grid floor of the shock chamber via a constant current scrambler circuit. During training sessions, each rat was placed in the safe compartment with its head facing away from the door. After $10 \mathrm{~s}$, the door separating the compartments was automatically opened, allowing the rat access to the shock compartment; the rats usually enter the shock (dark) compartment within 10-20 s of the door opening. As soon as rats stepped into the shock compartment a mild footshock was delivered. $(2 \mathrm{~s}, 0.60 \mathrm{~mA})$. For the western blot experiment (Fig. $1 \mathrm{~g}$ ) using IA extracts, animals were euthanized $30 \mathrm{~min}$ after training using halothane and their brains dissected. Dorsal hippocampi from trained animals were compared with dorsal hippocampi obtained from naive controls (animals that remained in their home cages)

Novel Object Location (NOL). For both mice and rats experiments, animals were allowed to familiarize with the arena for $5 \mathrm{~min}$ each day for three consecutive days before training. The arena consisted of a opaque box $(44.4 \mathrm{~cm} \times 44.4 \mathrm{~cm} \times 31.5 \mathrm{~cm}$ for rats and $28 \mathrm{~cm} \times 28 \mathrm{~cm} \times 20 \mathrm{~cm}$ for mice). Arena was placed in a room with a low level of light and sound-proof. During training session two identical objects $\left(\right.$ Lego $\left.{ }^{\circ}\right)$ were placed into the arena side by side and animals were allowed to freely explore them for $10 \mathrm{~min}$, returning to their home cages afterwards. During testing animals were placed back into the arena for 5 min and one object was moved to a different location which was counterbalanced between animals. Object exploration was defined as the orientation of the animal's nose towards the object at a distance $\leq 2 \mathrm{~cm}$ or as the animal placing its forepaws on the object; climbing on the object was not considered exploration. The objects and the arena were cleaned with $70 \%$ ethanol between animals to avoid olfactory cues. For NOL experiments with rats the sessions were videotaped and scored by an experimenter blind to experimental conditions; for NOL experiments with mice the sessions were scored using Ethovision (Noldus Information Technology). Memory retention was measured as $\%$ Preference calculated as the time spent exploring the object in the new location $(N)$ relative to the total exploration time $(N+$ familiar $(F))(\%$ Preference $=$ $\left(N /(N+F)^{\star} 100\right)^{70}$

Open field. For the locomotion experiment in rats, animals were allowed to freely explore for $5 \mathrm{~min}$ an open field arena $(44.4 \mathrm{~cm} \times 44.4 \mathrm{~cm} \times 31.5 \mathrm{~cm})$ divided into 16 imaginary quadrants. Locomotion was calculated as total number of crossings in the open field. An observer blind to experimental procedures scored the experiments. For mice, they were allowed to explore an empty arena $(34 \mathrm{~cm} \times 34 \mathrm{~cm} \times$ $23 \mathrm{~cm}$ ) for $10 \mathrm{~min}$ during which the total distance traveled as well as the time spent in the center or periphery of the arena were recorded using a video tracking system (Ethovision, Noldus Information Technology).

Spontaneous alternation and reversal learning in a $\mathbf{Y}$ maze. Spontaneous alternation and reversal learning were performed as described previously ${ }^{51}$. Briefly the Y-maze consisted of three white opaque arms (Med Associates) with sliding doors at the entrance of each arm. During spontaneous alternation test animals were allowed to freely explore the three arms from the center of the maze for $10 \mathrm{~min}$ and spontaneous alternation was defined as successive entries into each of the arms on overlapping triplets sets (e.g., $\mathrm{ABC}, \mathrm{BCA}, \mathrm{CAB}$, etc). The percentage of alternation was calculated by as the ratio of total alternations to possible alternation (total arm entries -2$) \times 100$. For the reversal learning experiment mice were single housed, food restricted and monitored daily until they reached $85 \%$ of their original weight before starting the experiment and during testing. They were given $1 / 2$ food pellet (LabDiet 5053) and one fruit loop (Kellog's) each day. The habituation phase was identical to spontaneous alternation. During the acquisition phase, one arm of the maze was chosen as the "correct arm" and baited with half of a fruit loop. The animals were initially restrained in the "start arm" for $1 \mathrm{~min}$ and then allowed to explore between the two arms. The acquisition phase consisted of 10 consecutive trials per day for 2 days (each day divided in 2 blocks of 5 trials each). Memory was calculated as the percentage of correct choice over each block of trials. During the reversal learning phase the "correct arm" was switched. The "correct arm" was counterbalanced between animals. Both experiments were scored by an observer blind to the experimental conditions and analyzed manually.

Marble burying test. Regular rat cages were used and filled with $\sim 5 \mathrm{~cm}$ deep bedding tamped down to make a flat, even surface. A regular pattern of 20 glass marbles was positioned on the surface of the bedding, spaced regularly, about $4 \mathrm{~cm}$ apart one from the other. Each animal was left in the cage for $30 \mathrm{~min}$ and the \% marbles buried was calculated as the number of marbles buried to $\sim 2 / 3$ of their depth over the total number of marbles $\times 100$.

Elevated plus maze. The elevated plus maze consisted of black Plexiglass fitted with white bottom surfaces to provide contrast and was placed $60 \mathrm{~cm}$ above the floor. The four arms ( 2 open and 2 closed) were interconnected by a central platform. Mice were placed at the center of the maze and were allowed to freely explore it for $5 \mathrm{~min}$ under red-lighting conditions. Time that each animal spent in the open and closed arms as well as the number of entries in the closed and open arms were recorded and further analyzed using Ethovision (Noldus Information Technology).

Plantar test (Hargreaves method). To assess mice nociceptive response, animals were placed in a clear plastic chamber $(45 \mathrm{~cm} \times 40 \mathrm{~cm}$, divided in 12 small animal enclosures, IITC Life Science) with a glass floor and allowed to acclimatize to the room and to the apparatus for $2 \mathrm{~h}$. After the acclimation period, the radiant heat source (infrared beam) was positioned under the glass floor directly beneath one of the animal's hind paws. The radiant heat source creates a $4 \times 6 \mathrm{~mm}$ intense spot on 
the paw. The paw withdrawal latency was determined using an electronic stopwatch coupled to the infrared source that switches off when the animal feels discomfort and withdraws its paw; a cutoff of $20 \mathrm{~s}$ for paw withdrawal was set up.

Electrophysiology. Field recording: Male Sprague-Dawley rats (6-8 weeks old) or $\sim 3$ months old mice (either Control or Wt1 1 mice) were deeply anesthetized with isoflurane and decapitated. The brain was rapidly removed and chilled in ice-cold artificial cerebro spinal fluid (ACSF) containing (in mM) $118 \mathrm{NaCl}, 3.5 \mathrm{KCl}, 2.5$ $\mathrm{CaCl}_{2}, 1.3 \mathrm{MgSO}_{4}, 1.25 \mathrm{NaH}_{2} \mathrm{PO}_{4}, 24 \mathrm{NaHCO}_{3}$, and 15 glucose, bubbled with $95 \%$ $\mathrm{O}_{2} / 5 \% \mathrm{CO}_{2}$. Transverse slices of dorsal hippocampus ( $400 \mu \mathrm{m}$ thick) were made on a tissue chopper at $4{ }^{\circ} \mathrm{C}$, and then placed in an interface chamber (ACSF and humidified $95 \% \mathrm{O}_{2} / 5 \% \mathrm{CO}_{2}$ atmosphere), where they were maintained at room temperature for at least $2 \mathrm{~h}$. For recording, slices were transferred to a submersion chamber and superfused with ACSF at $31 \pm 1{ }^{\circ} \mathrm{C}$. Monophasic, constant-current stimuli $(100 \mu \mathrm{s})$ were delivered with a bipolar stainless steel electrode positioned in stratum radiatum of area CA3, and field EPSPs (fEPSPs) were recorded in stratum radiatum of area CA1, using electrodes filled with ACSF $(\operatorname{Re}=2-4 \mathrm{M} \Omega)$. For all slices, initial spike threshold exceeded $2 \mathrm{mV}$. Signals were low-pass filtered at $3 \mathrm{kHz}$ and digitized at $20 \mathrm{kHz}$, and analyzed using pClamp 9 (Molecular Devices). Two HFS protocols were used: Weak-HFS, consisting of two trains separated by $20 \mathrm{~s}$, each consisting of 100 stimuli delivered at $100 \mathrm{~Hz}$ at an intensity that initially evoked a fEPSP measuring 20\% of spike threshold; and Strong-HFS, identical to Weak-HFS but delivered at an intensity that initially evoked a fEPSP of 75-80\% of spike threshold. For the LFS protocol, 900 pulses at $1 \mathrm{~Hz}$ were delivered at an intensity that initially evoked a fEPSP of $100 \%$ of spike threshold. In all experiments, the stimulation protocol was delivered at least $30 \mathrm{~min}$ after transfer of the slices to the recording chamber, when the basal fEPSP had been stable for at least $20 \mathrm{~min}$. Control slices were placed in the recording chamber and subjected only to test stimuli $(0.033 \mathrm{~Hz})$. Drug preincubations, when used, were performed at room temperature in submersion maintenance chambers containing ACSF saturated with bubbling $95 \% \mathrm{O}_{2} / 5 \% \mathrm{CO}_{2}$. Drugs were prepared as stock solutions and diluted to final concentrations in ACSF before use.

In slices where both the $\mathrm{TA} \rightarrow \mathrm{CA} 1$ and $\mathrm{SC} \rightarrow \mathrm{CA} 1$ inputs were activated stimulating electrodes were placed both in proximal stratum radiatum near the CA1/CA2 border (to activate Schaffer collaterals) and in the lacunosum moleculare within CA1 (to activate the perforant path). For the baseline period, slices were stimulated every $30 \mathrm{~s}$, alternating between Schaffer collaterals and perforant path. The perforant path was activated with theta-burst stimulation (TBS) consisting of 10 bursts at $5 \mathrm{~Hz}, 4$ pulses per burst at $100 \mathrm{~Hz}$, using $250 \mu \mathrm{A}$ stimuli. The Schaffer collaterals were stimulated with the same TBS pattern, delayed 20 ms delay relative to the perforant path, at an intensity that initially evoked $90 \%$ of the spike threshold. Recording electrodes were positioned in stratum radiatum and stratum lacunosum-moleculare. All slices had a spike threshold of at least $1.8 \mathrm{mV}$ in stratum radiatum.

For recordings in the presence of bicuculline, the brain was rapidly removed and chilled in ice-cold ACSF containing (in mM) $118 \mathrm{NaCl}, 2.5 \mathrm{KCl}, 4 \mathrm{CaCl}_{2}$ $4 \mathrm{MgSO}_{4}, 1.25 \mathrm{NaH}_{2} \mathrm{PO}_{4}, 24 \mathrm{NaHCO}_{3}$, and 15 glucose, bubbled with $95 \% \mathrm{O}_{2} / 5 \%$ $\mathrm{CO}_{2}$. Transverse slices of dorsal hippocampus $(400 \mu \mathrm{m}$ thick) were made on a tissue chopper at $4{ }^{\circ} \mathrm{C}$, and then placed in an interface chamber (ACSF and humidified $95 \% \mathrm{O}_{2} / 5 \% \mathrm{CO}_{2}$ atmosphere), where they were maintained at room temperature for at least $1 \mathrm{~h}$. The CA3 region was then dissected from CA1 region and slices were placed in a submersion chamber for $0.5-2.5 \mathrm{~h}$ before being transferred to the recording chamber. A Weak-HFS was delivered at a stimulus strength that evoked a fEPSP measuring $25-30 \%$ of spike threshold in bicuculline. All other conditions were as described above. Bicuculline was suspended in water to $10 \mathrm{mM}$ and diluted to $10 \mu \mathrm{M}$ in ACSF immediately before the experiment began.

Whole-cell recording: Adult male Sprague-Dawley rats (250-300 g) were deeply anesthetized with isoflurane and transcardially perfused with ice-cold ACSF. For experiments on excitability (Fig. 3f), the ACSF contained (in $\mathrm{mM}$ ): $\mathrm{NaCl}(128)$ D-glucose (10), $\mathrm{NaH}_{2} \mathrm{PO}_{4}(1.25), \mathrm{NaHCO}_{3}$ (25), $\mathrm{CaCl}_{2}$ (2), $\mathrm{MgSO}_{4}$ (2), and $\mathrm{KCl}$ (3), bubbled with $5 \% \mathrm{CO}_{2} / 95 \% \mathrm{O}_{2}(\mathrm{pH}=7.3,290-300 \mathrm{mOsM})$. Following perfusion, the brain was rapidly removed and chilled in ice-cold sucrose-ACSF containing (in $\mathrm{mM}$ ): sucrose (254), D-glucose (10), $\mathrm{NaH}_{2} \mathrm{PO} 4$ (1.25), $\mathrm{NaHCO}_{3}$ (25), $\mathrm{CaCl}_{2}$ (2), $\mathrm{MgSO}_{4}$ (2), and $\mathrm{KCl}(3)(\mathrm{pH}=7.3,290-310 \mathrm{mOsM})$. Coronal slices of dorsal hippocampus ( $200 \mu \mathrm{m}$ thick) were prepared using a vibratome in ice-cold sucroseACSF, and were allowed to recover submerged in bubbled ACSF for $45 \mathrm{~min}$ at $33 \pm$ $1{ }^{\circ} \mathrm{C}$, and thereafter at room temperature. Slices were transferred to a submersion recording chamber and perfused with $\operatorname{ACSF}(2 \mathrm{~mL} / \mathrm{min})$ at room temperature. CA1 pyramidal neurons were identified using IR DIC optics, and whole-cell recordings were obtained with an Axopatch 1D amplifier. Signals were low-pass filtered at $2 \mathrm{kHz}$ and digitized at $20 \mathrm{kHz}$, and no adjustment was made for pipette junction potential. Membrane excitability was tested in current clamp mode using pipettes containing (in $\mathrm{mM}$ ): $\mathrm{K}$ gluconate $(115), \mathrm{KCl}(20), \mathrm{MgCl}_{2}$ (1.5), phosphocreatineTris (10), Mg-ATP (2), Na-GTP (0.5), and Hepes (10) ( $\mathrm{pH}=7.3,280-285$ mOsM; 3.5-4.5 M $\Omega$ ). The membrane was depolarized with a series of ten $200 \mathrm{~ms}$-long current steps, increasing from 10 to $100 \mathrm{pA}$ from a holding potential of $-70 \mathrm{mV}$.

For recording spontaneous and miniature EPSCs (mEPSCs) (Supplementary Fig. 7a, b), slice preparation and recordings were performed in modified ACSF containing (in mM): $\mathrm{NaCl}$ (128), D-glucose (10), $\mathrm{NaH}_{2} \mathrm{PO}_{4}(1.25), \mathrm{NaHCO}_{3}(25)$,
$\mathrm{CaCl}_{2}$ (2), $\mathrm{MgCl}_{2}$ (2), and $\mathrm{KCl}(3)$ ( $\left.\mathrm{pH}=7.3,290-300 \mathrm{mOsM}\right)$, using pipettes filled with (in mM): Cs-methanesulfonate (130), HEPES (10), EGTA (0.5), $\mathrm{NaCl}(8)$, TEA-Cl (5), Mg-ATP (4), Na-GTP (0.4), Na-phosphocreatine (10), and N-ethyl lidocaine (1) $(\mathrm{pH}=7.3,280-285 \mathrm{mOsM} ; 3.0-4.5 \mathrm{M} \Omega)$. mEPSCs were recorded in the presence of $\mathrm{D}, \mathrm{L}-2$-amino-5-phosphonovaleric acid (APV; $50 \mu \mathrm{M})$, gabazine $(5 \mu \mathrm{M})$, and tetrodotoxin $(0.5 \mu \mathrm{M})$. Spontaneous events were recorded in the absence of inhibitors. 3-5 min after breakthrough, gap-free recordings were obtained for $10 \mathrm{~min}$. Only cells with stable input resistances $(<20 \%$ change as measured before and after the gap-free period) were included in the analysis. Template-based event detection was performed using Clampfit 10.3 (Molecular Devices). Templates were generated by averaging 5-10 events for each file, and the automated search results were verified manually.

Molecules and inhibitors used in electrophysiology. Bicuculline was purchased from Tocris (catalog \#2503) and resuspended in ACSF to reach a final concentration used $10 \mu \mathrm{M}$. The antibody against the IGF2 Receptor (IGF2-R Ab) was purchased from R\&D solutions (catalog \#AF2447) and used at a final concentration of $5 \mu \mathrm{g} / \mathrm{ml}$.

Transcriptomic profiling by mRNAseq. For the mRNAseq experiments, total RNA was extracted using Trizol (Thermo Fisher) from CAl regions isolated from rat hippocampal slices (Control vs LTP $90 \mathrm{~min}$ ). A pool of 10 CA1 regions collected from hippocampal slices of at least three different animals were necessary in order to obtain $\sim 1 \mu \mathrm{g}$ of total RNA for each condition. For the experiment relative to $W t 1 \Delta$ mice versus wild type littermates, dorsal hippocampus from naïve untrained animals were used. For the experiment relative to acute WT1 knockdown in rats (WT1-ODN vs Scrambled-ODN), dorsal hippocampus tissue surrounding the injection site was used. For all the mRNA sequencing experiments RNA integrity was checked by either the Agilent 2100 Bioanalyzer using the RNA 6000 Nano assay (Agilent, CA, USA). All processed total RNA samples had RIN value $\geq 9$. The seq library was prepared with the standard TruSeq RNA Sample Prep Kit v2 protocol (Illumina, CA, USA). Briefly, total RNA was poly-A-selected and then fragmented. The cDNA was synthesized using random hexamers, endrepaired and ligated with appropriate adapters for seq. The library then underwent size selection and purification using AMPure XP beads (Beckman Coulter, CA, USA). The appropriate Illumina-recommended $6 \mathrm{bp}$ barcode bases are introduced at one end of the adapters during PCR amplification step. The size and concentration of the RNAseq libraries was measured by the Agilent 2100 Bioanalyzer using the DNA 1000 assay (Agilent, CA, USA) before loading onto the sequencer The mRNA libraries were sequenced on the Illumina HiSeq 2000 System with 100 nucleotide single-end reads, according to the standard manufacturer's protocol (Illumina, CA, USA)

For the RNA-Seq data analysis Tophat $2.0 .13^{71}$, bowtie $2.1 .0^{72}$, samtool $0.1 .7^{73}$ and cufflinks $1.3 .0^{74}$ were used. The rn5-bowtie 2 index was generated with the command "bowtie2-build rn5.fa rn5". The "rn5.fa"-file was downloaded from the UCSC genome browser. The mm10-bowtie2 index was downloaded from http:// bowtie-bio.sourceforge.net/bowtie2/manual.shtml. RefSeq geneTracks and GTFfiles for the $\mathrm{rn} 5$ and $\mathrm{mm} 10$ genome assembly were downloaded from UCSC genome browser. Common gene ids in the GTF-files were matched to individual transcript_ids using the corresponding official symbols obtained from the geneTracks files.

The likelihood to detected a lowly to moderately expressed gene in a particular sample depends on the total number of sequenced reads, especially in case of lower reads counts $(<30,000,000)^{75}$. Therefore it could happen that more genes are detected in a sample with a higher read count than in a sample with a lower read count. This experimental artifact might distort normalization including total reads normalization as well as upper quartile normalization that is applied in this study. Both normalization methods only change the number of reads that are associated with a gene, but not the number of identified genes. In consequence, the same number of reads might be distributed over a different number of (by chance) experimentally identified genes in two samples, introducing gene expression differences between the two samples that do not exist. To prevent such experimental artifacts reads we applied an additional computational step before read alignment and differentially expressed genes detection. Under the assumption that during the seq process every fragment has the same chance to be sequenced, we ensured that each sample had the same number of total read counts by randomly removing reads from those samples with higher read counts than the minimum read count.

Reads were aligned to the rn5 or mm10 genome using Tophat with the option "--no-novel-juncs" and the refSeq-GTF-file (the option "--solexa1.3-quals" was additionally chosen in case of the rat samples). Differentially expressed genes were identified using Cuffdiff with the options "--upper-quartile-norm", "--fragbias-correct" against the rn5 genome and "--multi-read-correct" and the refSeqGTF-file.

In each analysis all differentially expressed genes (DEGs) that were statistically significant $(\mathrm{FDR}=5 \%)$ were considered. DEGs with a minimum fold change of $\log _{2}\left(\left(\mathrm{FPKM}_{\text {condition } 1}+1\right) /\left(\mathrm{FPKM}_{\text {condition } 2}+1\right)\right)>= \pm \log _{2}(1.3)$ were submitted to pathway enrichment analysis as described below. 
Analysis of transcriptomic data. Enrichment analysis using mRNA seq data was performed similarly as previously described ${ }^{76}$. The "Transfac and jaspar pwms" library was downloaded from the EnrichR website ${ }^{77}$. All human transcription factor gene associations were kept. Human target genes and transcription factors were replaced by their rat homologs based on the mouse informatics database (Mouse Genome Informatics, http://www.informatics.jax.org, 5/24/2013) and the National Center for Biotechnology Information homologene database (http://www. ncbi.nlm.nih.gov/homologene/, 06/01/2018). Mouse gene-transcription factor associates were removed from the database.

To increase the statistical accuracy we removed all gene symbols in both databases that are not part of the RefSeq rn5 gene annotation and therefore could not be identified as differentially expressed. Similarly, we removed all differentially expressed genes that were not part of the "Transfac_and_jaspar_pwms" library. Right tailed fisher's exact test was used for enrichment analysis and the negative logarithms to the basis 10 of the $p$-values were calculated.

Control theory-based toy model of WT1 function. Input of an experience to the hippocampus is represented as a rectangular pulse. Neuronal activity in the hippocampus converts this pulse into a more long lasting output with respect to the time scale of the experiments (days), which we represent as a time integrator. Thus, the area under the rectangular pulse becomes a step function as inputs to memorystrengthening and memory-weakening pathways. We are unaware of any experimental data to suggest reasonable values for the magnitude of this step input, $\underline{u}$, hence arbitrary values were chosen and $u$ was subsequently varied to make a range of predictions (Supplementary Fig. 7). W'e model memory-strengthening and memory-weakening signaling as two first order processes in parallel. A first order process is governed by the following equation:

$$
\tau \frac{d x}{d t}=-x+K \cdot u(t)
$$

Here, $\tau$ is the time constant, $K$ is the steady state gain, $u$ is the input strength, $t$ is time, and $x$ is the dependent variable (in this case memory-strengthening or memory-weakening signal strength). We denote memory-strengthening with the subscript 1 and memory-weakening with the subscript 2. Because activation of one cell's signaling could affect other non-activated cells, we take both gains $\left(K_{1}\right.$ and $K_{2}$ ), to be 3, reflecting signal amplification. However, in the model the effects of these gains and the input magnitude are indistinguishable, so our parameter variation exercise effectively explored both of these avenues. Additionally, we estimated from electrophysiological data that lack of functional WT1 induces an $\sim 2.4-$ fold increase in the input signal strength, so in the case of Wt1L mice, we take the gains as 7.2. The time constants $\tau_{1}$ and $\tau_{2}$ were tuned to be consistent with the data in Figs. 1-3. Thus, $\tau_{1}$ for memory-strengthening signaling was taken as fast $(0.5 \mathrm{~h})$ and not affected by lack of functional WT1, whereas $\tau_{2}$ for memory-weakening signaling was taken as slow $(36 \mathrm{~h})$ and took a different value for Wt1 $\Delta$ animals $(144 \mathrm{~h})$. These model parameters are summarized in the below Table 2:

The difference of these two process outputs was passed through a saturation function (based on neurobiological reasoning presented in the main text), to be fixed between 0 and 1, which we call "Pathway Activity". Thus,

$$
\text { Pathway Activity }=\operatorname{sat}\left(x_{1}-x_{2}, 0,1\right) .
$$

This "Pathway Activity" variable coarsely represents an amalgamated capacity for learning new events in the short-term. Based on the assumption of a finite amount of downstream effectors that interpret pathway activity, we define

$$
\text { Effectors Available }=1-\text { Pathway Activity. }
$$

We specify that "Memory" is a function of pathway and effectors dynamics by the following logic. In the absence of any past event, we can calculate the peak of Pathway Activity elicited by a particular event. This peak value is taken as the amount of capacity required to fully learn, which we call "need". Then, we can calculate the Effectors Available elicited by a particular event as a function of time, given that other events may have already occurred previously, which we call "have". Memory at each time point is defined as the Pathway Activity attributable to a particular event, divided by its maximum value, but weighted by the fraction have/ need. Specifically,

$$
\text { Memory }=\frac{(\text { Pathway Activity })_{i}}{\max (\text { Pathway Activity })_{i}} \frac{\text { have }_{i}}{\text { need }_{i}},
$$

where subscript $i$ here denotes a particular learning input event. Thus, if there were not enough "Effectors Available" at the time of an event's stimulus, have/need is reduced, and thus Memory is lowered.

All simulations were performed in MATLAB (The Mathworks, Natick, MA) and the code is available upon request.

Reporting summary. Further information on research design is available in the Nature Research Reporting Summary linked to this article.

\section{Data availability}

The data that support the findings of this study are available from the corresponding authors upon request. Raw and processed mRNAseq data was uploaded to GEO

Omnibus. Accessions code are:

-mRNA seq_ DEGs in LTP 90 min: GEO Series GSE120712

-mRNA seq_DEGs in Wt1 $\triangle$ mice: GEO Series GSE120708

\section{Code availability}

Custom written Matlab scripts were used for the control theory-based model of WT1 function. The code is available upon request.

Received: 30 October 2018 Accepted: 31 July 2019

Published online: 21 August 2019

\section{References}

1. McGaugh, J. L. Memory-a century of consolidation. Science 287, 248-251 (2000).

2. Alberini, C. M. \& Kandel, E. R. The regulation of transcription in memory consolidation. Cold Spring Harb. Perspect. Biol. 7, a021741 (2014).

3. Davis, R. L. \& Zhong, Y. The biology of forgetting-a perspective. Neuron $\mathbf{9 5}$, 490-503 (2017)

4. Abel, T. \& Kandel, E. Positive and negative regulatory mechanisms that mediate long-term memory storage. Brain Res. Brain Res. Rev. 26, 360-378 (1998).

5. Kandel, E. R. The molecular biology of memory: cAMP, PKA, CRE, CREB-1, CREB-2, and CPEB. Mol. Brain 5, 14 (2012).

6. Ahn, H. J. et al. c-Rel, an NF-kappaB family transcription factor, is required for hippocampal long-term synaptic plasticity and memory formation. Learn. Mem. 15, 539-549 (2008).

7. Alberini, C. M. Transcription factors in long-term memory and synaptic plasticity. Physiol. Rev. 89, 121-145 (2009).

8. Graff, J., Woldemichael, B. T., Berchtold, D., Dewarrat, G. \& Mansuy, I. M. Dynamic histone marks in the hippocampus and cortex facilitate memory consolidation. Nat. Commun. 3, 991 (2012).

9. Shuai, Y. et al. Dissecting neural pathways for forgetting in Drosophila olfactory aversive memory. Proc. Natl Acad. Sci. USA 112, E6663-E6672 (2015).

10. Liu, Y. et al. Hippocampal activation of Racl regulates the forgetting of object recognition memory. Curr. Biol. 26, 2351-2357 (2016).

11. Cervantes-Sandoval, I., Chakraborty, M., MacMullen, C. \& Davis, R. L. Scribble scaffolds a signalosome for active forgetting. Neuron 90, 1230-1242 (2016).

12. Himmelreich, S. et al. Dopamine receptor DAMB signals via Gq to mediate forgetting in Drosophila. Cell Rep. 21, 2074-2081 (2017).

13. Migues, P. V. et al. Blocking synaptic removal of GluA2-containing AMPA receptors prevents the natural forgetting of long-term memories. J. Neurosci. Off. J. Soc. Neurosci. 36, 3481-3494 (2016).

14. Akers, K. G. et al. Hippocampal neurogenesis regulates forgetting during adulthood and infancy. Science 344, 598-602 (2014).

15. Abel, T. et al. Genetic demonstration of a role for PKA in the late phase of LTP and in hippocampus-based long-term memory. Cell 88, 615-626 (1997).

16. Bourtchuladze, R. et al. Deficient long-term memory in mice with a targeted mutation of the cAMP-responsive element-binding protein. Cell 79, 59-68 (1994).

17. Pena de Ortiz, S., Maldonado-Vlaar, C. S. \& Carrasquillo, Y. Hippocampal expression of the orphan nuclear receptor gene hzf-3/nurrl during spatial discrimination learning. Neurobiol. Learn. Mem. 74, 161-178 (2000)

18. Taubenfeld, S. M., Milekic, M. H., Monti, B. \& Alberini, C. M. The consolidation of new but not reactivated memory requires hippocampal C/ EBPbeta. Nat. Neurosci. 4, 813-818 (2001).

19. Schoch, H. \& Abel, T. Transcriptional co-repressors and memory storage. Neuropharmacology 80, 53-60 (2014).

20. Abel, T., Martin, K. C., Bartsch, D. \& Kandel, E. R. Memory suppressor genes: inhibitory constraints on the storage of long-term memory. Science $\mathbf{2 7 9}$, 338-341 (1998)

21. Yin, J. C., Del Vecchio, M., Zhou, H. \& Tully, T. CREB as a memory modulator: induced expression of a dCREB2 activator isoform enhances longterm memory in Drosophila. Cell 81, 107-115 (1995).

22. Bartsch, D., Casadio, A., Karl, K. A., Serodio, P. \& Kandel, E. R. CREB1 encodes a nuclear activator, a repressor, and a cytoplasmic modulator that form a regulatory unit critical for long-term facilitation. Cell 95, 211-223 (1998).

23. Adachi, M., Autry, A. E., Covington, H. E. 3rd \& Monteggia, L. M. MeCP2mediated transcription repression in the basolateral amygdala may underlie 
heightened anxiety in a mouse model of Rett syndrome. J. Neurosci.: Off. J. Soc. Neurosci. 29, 4218-4227 (2009).

24. Carrion, A. M., Link, W. A., Ledo, F., Mellstrom, B. \& Naranjo, J. R. DREAM is a Ca2+-regulated transcriptional repressor. Nature 398, 80-84 (1999).

25. Cole, C. J. et al. MEF2 negatively regulates learning-induced structural plasticity and memory formation. Nat. Neurosci. 15, 1255-1264 (2012).

26. Lynch, M. A. Long-term potentiation and memory. Physiol. Rev. 84, 87-136 (2004).

27. Rauscher, F. J. 3rd The WT1 Wilms tumor gene product: a developmentally regulated transcription factor in the kidney that functions as a tumor suppressor. FASEB J. 7, 896-903 (1993).

28. Fischbach, B. V., Trout, K. L., Lewis, J., Luis, C. A. \& Sika, M. WAGR syndrome: a clinical review of 54 cases. Pediatrics 116, 984-988 (2005).

29. Lovell, M. A., Xie, C., Xiong, S. \& Markesbery, W. R. Wilms' tumor suppressor (WT1) is a mediator of neuronal degeneration associated with the pathogenesis of Alzheimer's disease. Brain Res. 983, 84-96 (2003).

30. von Schimmelmann, M. et al. Polycomb repressive complex 2 (PRC2) silences genes responsible for neurodegeneration. Nat. Neurosci. 19, 1321-1330 (2016).

31. Ladomery, M. R., Slight, J., Mc Ghee, S. \& Hastie, N. D. Presence of WT1, the Wilm's tumor suppressor gene product, in nuclear poly $(\mathrm{A})(+)$ ribonucleoprotein. J. Biol. Chem. 274, 36520-36526 (1999).

32. Morrison, A. A., Venables, J. P., Dellaire, G. \& Ladomery, M. R. The Wilms tumour suppressor protein WT1 (+KTS isoform) binds alpha-actinin 1 mRNA via its zinc-finger domain. Biochem. Cell Biol. 84, 789-798 (2006).

33. Uschkereit, C. et al. Different CTNNB1 mutations as molecular genetic proof for the independent origin of four Wilms tumours in a patient with a novel germ line WT1 mutation. J. Med. Genet. 44, 393-396 (2007).

34. Grover, L. M. \& Yan, C. Blockade of GABAA receptors facilitates induction of NMDA receptor-independent long-term potentiation. J. Neurophysiol. 81, 2814-2822 (1999).

35. Magee, J. C. \& Johnston, D. A synaptically controlled, associative signal for Hebbian plasticity in hippocampal neurons. Science 275, 209-213 (1997).

36. Basu, J. \& Siegelbaum, S. A. The corticohippocampal circuit, synaptic plasticity, and memory. Cold Spring Harb. Perspect. Biol. 7, a021733 (2015).

37. Judge, S. J. \& Hasselmo, M. E. Theta rhythmic stimulation of stratum lacunosum-moleculare in rat hippocampus contributes to associative LTP at a phase offset in stratum radiatum. J. Neurophysiol. 92, 1615-1624 (2004).

38. Costa, R., Goncalves, A., Saraiva, M. J. \& Cardoso, I. Transthyretin binding to A-Beta peptide-impact on A-Beta fibrillogenesis and toxicity. FEBS Lett. 582, 936-942 (2008).

39. Monopoli, M. P. et al. Temporal proteomic profile of memory consolidation in the rat hippocampal dentate gyrus. Proteomics 11, 4189-4201 (2011).

40. Kang, J. et al. Radiation-induced overexpression of transthyretin inhibits retinol-mediated hippocampal neurogenesis. Sci. Rep. 8, 8394 (2018).

41. Vieira, M., Gomes, J. R. \& Saraiva, M. J. Transthyretin induces insulin-like growth factor I nuclear translocation regulating its levels in the hippocampus. Mol. Neurobiol. 51, 1468-1479 (2015).

42. Drummond, I. A. et al. Repression of the insulin-like growth factor II gene by the Wilms tumor suppressor WT1. Science 257, 674-678 (1992).

43. $\mathrm{Hu}$, Q. et al. Wt1 ablation and Igf2 upregulation in mice result in Wilms tumors with elevated ERK1/2 phosphorylation. J. Clin. Investig. 121, 174-183 (2011).

44. Chen, D. Y. et al. A critical role for IGF-II in memory consolidation and enhancement. Nature 469, 491-497 (2011).

45. Stern, S. A., Chen, D. Y. \& Alberini, C. M. The effect of insulin and insulin-like growth factors on hippocampus- and amygdala-dependent long-term memory formation. Learn. Mem. 21, 556-563 (2014).

46. Maren, S. \& Quirk, G. J. Neuronal signalling of fear memory. Nat. Rev. Neurosci. 5, 844-852 (2004).

47. Lalonde, R. The neurobiological basis of spontaneous alternation. Neurosci. Biobehav Rev. 26, 91-104 (2002).

48. Rampon, C. et al. Effects of environmental enrichment on gene expression in the brain. Proc. Natl Acad. Sci. USA 97, 12880-12884 (2000).

49. Martinez, G. et al. Regulation of memory formation by the transcription factor XBP1. Cell Rep. 14, 1382-1394 (2016).

50. Awasthi, A. et al. Synaptotagmin-3 drives AMPA receptor endocytosis, depression of synapse strength, and forgetting. Science 363, eaav1483 (2019).

51. Stern, S. A., Kohtz, A. S., Pollonini, G. \& Alberini, C. M. Enhancement of memories by systemic administration of insulin-like growth factor II. Neuropsychopharmacology 39, 2179-2190 (2014).

52. Agis-Balboa, R. C. et al. A hippocampal insulin-growth factor 2 pathway regulates the extinction of fear memories. EMBO J. 30, 4071-4083 (2011)

53. Fernandes, D. \& Carvalho, A. L. Mechanisms of homeostatic plasticity in the excitatory synapse. J. Neurochem. 139, 973-996 (2016)

54. Shuai, Y. et al. Forgetting is regulated through Rac activity in Drosophila. Cell 140, 579-589 (2010).

55. Berry, J. A., Cervantes-Sandoval, I., Chakraborty, M. \& Davis, R. L. Sleep facilitates memory by blocking dopamine neuron-mediated forgetting. Cell 161, 1656-1667 (2015).
56. Dong, Z. et al. Long-term potentiation decay and memory loss are mediated by AMPAR endocytosis. J. Clin. Investig. 125, 234-247 (2015).

57. Shepherd, J. D. et al. Arc/Arg3.1 mediates homeostatic synaptic scaling of AMPA receptors. Neuron 52, 475-484 (2006).

58. Aoto, J., Nam, C. I., Poon, M. M., Ting, P. \& Chen, L. Synaptic signaling by alltrans retinoic acid in homeostatic synaptic plasticity. Neuron 60, 308-320 (2008)

59. Kang, J. X., Bell, J., Leaf, A., Beard, R. L. \& Chandraratna, R. A. Retinoic acid alters the intracellular trafficking of the mannose-6-phosphate/insulin-like growth factor II receptor and lysosomal enzymes. Proc. Natl Acad. Sci. USA 95, 13687-13691 (1998)

60. Whitlock, J. R., Heynen, A. J., Shuler, M. G. \& Bear, M. F. Learning induces long-term potentiation in the hippocampus. Science 313, 1093-1097 (2006).

61. Habib, D., Tsui, C. K., Rosen, L. G. \& Dringenberg, H. C. Occlusion of lowfrequency-induced, heterosynaptic long-term potentiation in the rat hippocampus in vivo following spatial training. Cereb. Cortex 24, 3090-3096 (2014).

62. Rioult-Pedotti, M. S., Friedman, D. \& Donoghue, J. P. Learning-induced LTP in neocortex. Science 290, 533-536 (2000).

63. Cantarero, G., Tang, B., O’Malley, R., Salas, R. \& Celnik, P. Motor learning interference is proportional to occlusion of LTP-like plasticity. J. Neurosci.: Off. J. Soc. Neurosci. 33, 4634-4641 (2013).

64. Hodgson, R. A. et al. Training-induced and electrically induced potentiation in the neocortex. Neurobiol. Learn. Mem. 83, 22-32 (2005).

65. Parsons, R. G. \& Ressler, K. J. Implications of memory modulation for posttraumatic stress and fear disorders. Nat. Neurosci. 16, 146-153 (2013).

66. Kaifosh, P. \& Losonczy, A. Mnemonic functions for nonlinear dendritic integration in hippocampal pyramidal circuits. Neuron 90, 622-634 (2016).

67. Gao, F. et al. The Wilms tumor gene, Wt1, is required for Sox9 expression and maintenance of tubular architecture in the developing testis. Proc. Natl Acad. Sci. USA 103, 11987-11992 (2006).

68. Tsien, J. Z. et al. Subregion- and cell type-restricted gene knockout in mouse brain. Cell 87, 1317-1326 (1996).

69. Menke, A. L. et al. The wt1-heterozygous mouse; a model to study the development of glomerular sclerosis. J. Pathol. 200, 667-674 (2003).

70. Antunes, M. \& Biala, G. The novel object recognition memory: neurobiology, test procedure, and its modifications. Cogn. Process. 13, 93-110 (2012).

71. Trapnell, C., Pachter, L. \& Salzberg, S. L. TopHat: discovering splice junctions with RNA-Seq. Bioinformatics 25, 1105-1111 (2009).

72. Langmead, B. \& Salzberg, S. L. Fast gapped-read alignment with Bowtie 2. Nat Methods 9, 357-359 (2012).

73. $\mathrm{Li}, \mathrm{H}$. et al. The sequence alignment/map format and SAMtools Bioinformatics 25, 2078-2079 (2009).

74. Trapnell, C. et al. Transcript assembly and quantification by RNA-Seq reveals unannotated transcripts and isoform switching during cell differentiation. Nat. Biotechnol. 28, 511-515 (2010).

75. McIntyre, L. M. et al. RNA-seq: technical variability and sampling. $B M C$ Genom. 12, 293 (2011).

76. Karakikes, I. et al. Small molecule-mediated directed differentiation of human embryonic stem cells toward ventricular cardiomyocytes. Stem Cells Transl. Med. 3, 18-31 (2014).

77. Kuleshov, M. V. et al. Enrichr: a comprehensive gene set enrichment analysis web server 2016 update. Nucleic Acids Res. 44, W90-W97 (2016).

\section{Acknowledgements}

The authors thank P.Y. Chuang for his guidance with the Wt1 $\Delta$ mice colony and G. Jayaraman for her assistance with biochemical experiments. The authors want to thank Rachael L. Neve at MIT Viral Core for her assistance in generating the HSV viruses. This research was supported by NIH grants GM54508 and Systems Biology Center Grant GM071558 (PI: Iyengar), R37- MH065635 (PI: Alberini). The authors aknowledge the Elsevier Publishing Group for the permission granted to use Figure 36 from the Rat Brain in Stereotaxic Coordinates, by George Paxinos, Charles Watson, Copyright Elsevier, 4th Edition (1998) now represented in Fig. 2c, d.

\section{Author contributions}

The project was designed by R.I., C.M., L.M., R.D.B., C.M.A. C.M. conducted most of the biochemical experiments, the surgeries in rats and the behavior experiments in rats. L.M. conducted all the behavior experiments in mice. E.G., J.M.S. and A.S. performed field recordings experiments while N.T. conducted patch clamp experiments. J.H. analyzed mRNAseq data and did enrichment analysis. S.A.S. and V.G. helped with inhibitory avoidance and contextual fear conditioning experiments in rats. G.E.H. and S.J.R. provided equipment and guidance for mice behavioral experiments. H.A. designed and assisted with the cloning strategy for WT1-HSV viruses. E.U.A. helped with microscopy V.H. provided the $W t 1^{f l f l}$ animals and provided expert insight in WT1 function and genetic manipulation. M.R.B. developed and ran the memory homeostasis model. C.M., L.M., R.D.B., C.M.A. and R.I. wrote the manuscript. All authors assisted in editing the manuscript. 


\section{Additional information}

Supplementary Information accompanies this paper at https://doi.org/10.1038/s41467019-11781-x.

Competing interests: The authors declare no competing interests.

Reprints and permission information is available online at http://npg.nature.com/ reprintsandpermissions/

Peer review information: Nature Communications thanks Daniel Storm and the other, anonymous, reviewer(s) for their contribution to the peer review of this work.

Publisher's note: Springer Nature remains neutral with regard to jurisdictional claims in published maps and institutional affiliations. (c) (i) Open Access This article is licensed under a Creative Commons Attribution 4.0 International License, which permits use, sharing, adaptation, distribution and reproduction in any medium or format, as long as you give appropriate credit to the original author(s) and the source, provide a link to the Creative Commons license, and indicate if changes were made. The images or other third party material in this article are included in the article's Creative Commons license, unless indicated otherwise in a credit line to the material. If material is not included in the article's Creative Commons license and your intended use is not permitted by statutory regulation or exceeds the permitted use, you will need to obtain permission directly from the copyright holder. To view a copy of this license, visit http://creativecommons.org/ licenses/by/4.0/.

(C) The Author(s) 2019 